RUNNING HEAD: EXTRACTING INFORMATION FROM FACES

Is that Clint Eastwood or an old person? Assessing the speed of extracting group versus person information from faces

Daniel P. Skorich ${ }^{1,2}$, Kenneth I. Mavor ${ }^{3}$, S. Alexander Haslam² ${ }^{2}$ and Joel L. Larwood ${ }^{2}$

\author{
${ }^{1}$ Research School of Psychology, Australian National University \\ ${ }^{2}$ School of Psychology, University of Queensland \\ ${ }^{3}$ School of Psychology and Neuroscience, University of St Andrews
}

Address for correspondence:

Daniel Skorich

Research School of Psychology,

Building 39, Science Road

The Australian National University

Acton, ACT, 2601, Australia

Phone: + 61261252796

Email: Daniel.Skorich@anu.edu.au

Acknowledgements. This research was supported by the Australian Research Council (FLFL110100199) and the Canadian Institute for Advanced Research (Social Interactions Identity and Well-Being Program). 


\begin{abstract}
The human face is a key source of social information. In particular, it communicates a target's personal identity and some of their main group memberships. Different models of social perception posit distinct stages at which this group-level and person-level information is extracted from the face, with divergent downstream consequences for cognition and behavior. This paper presents four experiments that explore the time-course of extracting group and person information from faces. In Experiments 1 and 2, we explore the effect of chunked versus unchunked processing on the speed of extracting group versus person information, as well as the impact of familiarity in Experiment 2. In Experiment 3, we examine the effect of the availability of a diagnostic cue on these same judgments. In Experiment 4, we explore the effect of both group-level and person-level prototypicality of face exemplars. Across all four experiments, we find no evidence for the perceptual primacy of either group or person information. Instead, we find that chunked processing, featural processing based on a single diagnostic cue, familiarity, and the prototypicality of face exemplars all result in a processing speed advantage for both group-level and person-level judgments equivalently. These results have important implications for influential models of face processing and impression formation, and can inform — and be integrated with — an understanding of the process of social categorization more broadly.
\end{abstract}

Keywords: face processing; categorization; individuation; identification; stereotyping. 


\section{Is that Clint Eastwood or an old person? Assessing the speed of extracting group versus person information from faces.}

\section{Introduction}

The human face is an important source of social information. It allows perceivers to infer the mental states and behavioral responses of others (Baron-Cohen, 2005; Baron-Cohen, Jolliffe, Mortimer \& Robertson, 1997) and to keep track of both their stable and shifting social relations with others (Macrae, Quinn, Mason, \& Quadflieg, 2005). Specifically, the face provides cues to a target person's focus of attention, their cognitive and emotional states, their personal identity, and some of the key social groups to which they belong. Together, this helps perceivers to successfully navigate their social worlds. Yet different models of social perception disagree about the stage at which this information is extracted from the face, thereby positing different downstream consequences for impressions of the target and for behavior towards them. For this reason, it is important to establish the order in which a perceiver extracts different types of information from faces. In the current paper, we explore particularly the speed and ease of extracting group versus person information from faces. We report four experiments in which we investigate how the processing strategy, the presence or absence of diagnostic cues, the familiarity of the perceiver with the individual identity or group membership of a face, and the prototypicality of face exemplars impacts on the ease of extracting group and person information from faces.

\subsection{Group and Person Information in Faces: Theoretical Accounts}

The human face provides a diverse array of information to other perceivers, which enables and facilitates social interaction (Bruce \& Young, 2012). Through its variable, context-dependent expressions, the face provides information about a target's current focus of attention and their cognitive and emotional state (Palermo et al., 2018). Through its relatively stable and enduring physical characteristics, the face provides information about a target's 
personal identity, their longer-term personality traits, and some of their group memberships — in particular their sex, age, and ethnic background (Todorov, Olivola, Dotsch \& MendeSiedlecki, 2015). Person and group information in faces — that is, a target's personal identity and their group memberships, respectively — is extracted from similar areas of the face (Burton, Jenkins, Hancock \& White, 2005), and, with training, the extraction of both types of information can become relatively easy and relatively automatic (Blair, Judd \& Fallman, 2004; Yan, Young \& Andrews, 2017). Despite this apparent similarity in ease and automaticity of extraction, two prominent models in this area - Bruce and Young's (1986) face recognition model and Fiske and Neuberg's (1990) continuum model of impression formation — provide different accounts of perceptual primacy for the extraction of group and person information, with important downstream consequences for social perception and behavior.

Bruce and Young (1986; Young and Bruce, 2011) propose that face recognition is a sequential process, consisting of a series of distinct stages. Each of these stages is argued to be supported by different cognitive processes, which themselves recruit a number of different 'codes' (or types of information) for their functioning. In focusing on the face recognition components of the model that are most relevant to the current research (and ignoring those concerned with emotion recognition and facial speech analysis), we highlight five codes of interest. First, the pictorial code consists of a description of an individual photo or instance of a face and is fundamental to the interpretation of incoming face exemplars. Second, the structural code consists of a coherent representation of an individual face, from which variable information such as changes in angle, lighting, expression and so forth has been subtracted. Third, the visually derived semantic code consists of information that is derived directly from the physical characteristics of the face, including the personal identity and group memberships of the person whose face it is. Fourth, the identity-specific semantic code 
consists of information that can be inferred about a face once it has been identified - that is, information that is associated in memory with the person whose face it is, such as their profession, what car they drive, their likes and dislikes. Finally, the name code represents the name attached to the face, by which we denote who a person is in common language.

According to Bruce and Young's (1986) face recognition model, the individual identity of a face is extracted directly from the physical characteristics of the face, via the visually derived semantic code. Following structural encoding of a face, which occurs very early in the processing stream, the physical characteristics of the resulting face representation allow a perceiver to determine if the face belongs to a person previously known to them or not and, if it does, to recognize that person as familiar. After this familiarity judgment, a perceiver can then identify the person specifically (if, again, the person is known to them), after which they can access semantic information associated with them, via the identityspecific semantic code, as well as their name via the name code.

Similarly, Bruce and Young (1986) suggest that certain group memberships, like individual identity, can be extracted directly from the physical characteristics of the face, also through the visually derived semantic code. These group memberships include sex, age, and ethnic identity, because each of them is evident on the basis of visually available cues in the face — with more recent evidence suggesting that sexual orientation (Rule, Ambady \& Hallett, 2009) and even perhaps political affiliation (Peterson, Jacobs, Hibbing \& Smith, 2018; Rule \& Sutherland, 2017) might also be visually available. Importantly, however, Bruce and Young's model also suggests that information about (some) group memberships can be extracted from the face after the face has been identified, via the identity-specific semantic code. That is, a face's sex, age, ethnic identity and other group memberships can be inferred on the basis of identifying to whom a face belongs, because a perceiver may have encoded a target's group memberships alongside their individual identity (Rossion, 2002). 
The Bruce and Young (1986) face recognition model therefore suggests that both person and group information is relatively easy to extract from a face, and that person information is either extracted earlier in the processing stream, or at the same time as group information (Bruce, Ellis, Gibling \& Young, 1987).

In contrast to Bruce and Young's (1986) face recognition model, the continuum model of impression formation (Fiske \& Neuberg, 1990; Fiske, Lin \& Neuberg, 1999) suggests that group information is easier to process than person information, and therefore that it is employed earlier in the processing stream than person information. In keeping with the motivated tactician perspective (Fiske \& Taylor, 1991) in which their model is embedded, Fiske and Neuberg (1990) argue that person perception proceeds through a series of stages, from more group-based representations at the early stages through ever more person-based representations at the latter stages, as a function of the motivated selective deployment of cognitive resources. These researchers argue that the initial (automatic) categorization of the target of perception will be at the group-level, because the group memberships of sex, age, and ethnic identity are easily discerned from visual cues in the face. They further argue that it is only after resource-depleting cognitive processing of available information about a target that person-level information and personal identity will be constructed and engaged. The continuum model of impression formation therefore suggests that group information should be easier to discern than person information, and that group memberships will be invoked more quickly, and earlier in the processing stream, than personal identity - in direct contrast to Bruce and Young's (1986) face recognition model.

\subsection{Group and Person Information in Faces: Empirical Evidence}

Despite these well-developed theoretical accounts, empirical research directly comparing the extraction of group and person information from faces is thin on the ground. Evidence from event-related potential (ERP) studies suggest that race, sex, age and individual 
identity can all be extracted from faces as early as $100-200 \mathrm{~ms}$ after stimulus exposure (Ito \& Urland, 2003; 2005; Senholzi \& Ito, 2013), with activation in the face-specific N170 component often observed (Bentin \& Deouell, 2000; Eimer, 2000a; Freeman, Ambady \& Holcomb, 2010; Huang et al., 2017), as well as in other later components such as the N400, which has often been associated with semantic discrimination (Caldara, Jermann, Arango, \& Van der Linden, 2004; Hehman, Volpert, \& Simons, 2014). Together this research provides some hints that person and group information are equally easy to extract from faces, as suggested by Bruce and Young's (1986) face recognition model, and in contrast to the continuum model of impression formation.

In the only set of experiments directly comparing the extraction of group and person information from faces, Cloutier and his colleagues (Cloutier \& Macrae, 2007; Cloutier, Mason \& Macrae, 2005) have found, however, that group information is extracted earlier and more easily than person information. Cloutier et al. (2005), across two experiments, investigated the speed of judgments of sex and judgments of identity, and the moderating role of facial inversion and facial blurring on these judgments. Subsequently, Cloutier and Macrae (2007) also compared the speed of sex and identity judgments, but this time examining the moderating role of facial rotation. In each of these experiments, participants were presented with a number of faces, one-by-one. For each face, the participant was asked to respond as quickly and as accurately as possible to indicate whether the face was male or female (in the sex group membership blocks) or that of a familiar or unfamiliar person (in the individual identity blocks). In the sex blocks, all photos were of unfamiliar people, half of whom were male and half of whom were female. In the identity blocks, half the photos were of equal numbers of well-known male and female celebrities (of the late 1990's and early 2000s, e.g., Britney Spears, Bruce Willis, David Duchovny), and half were of equal numbers of male and female strangers. In Cloutier et al.'s (2005) experiments, half the faces were presented 
upright (Experiment 1) or unblurred (Experiment 2), and half were presented upside-down (Experiment 1) or blurred (Experiment 2). In Cloutier and Macrae's (2007) experiment, each face was presented, within-subjects, in one of five orientations, ranging from $0^{\circ}$ (upright) to $180^{\circ}$ (upside-down). Response accuracy and latency were recorded.

Cloutier et al. (2005) found that participants were faster at responding to indicate that a face was male or female than they were at responding to indicate that a face belonged to a familiar or unfamiliar person, irrespective of facial inversion or blurring. They also found that judgments of sex group membership appeared to be less affected by inversion and blurring than were judgments of identity. Replicating the results of Cloutier et al. (2005), Cloutier and Macrae (2007) found a main effect difference between judgments of sex group membership and judgments of identity, irrespective of the angle of rotation. Similar to Cloutier et al., (2005) they also found that sex group membership judgments were more robust to the angle of rotation than were personal identity judgments. All these experiments suggest that group information is easier to extract from faces than person information, and that it occurs earlier in the processing stream, as argued by the continuum model of impression formation (Fiske \& Neuberg, 1990), and by the motivated tactician perspective (Fiske \& Taylor, 1991) in which that model is theoretically embedded.

In explaining these results, Cloutier and colleagues $(2005,2007)$ suggest that group membership information is extracted from faces more quickly and earlier in the processing stream than personal identity because doing so invokes heuristic operations that are fundamentally simpler. They further argue that the extraction of group membership information, in contrast to person information, is facilitated by the presence of simple diagnostic cues. Specifically, they argue that participants are able to use hair length as a simple cue for making their sex group membership decisions, because all the male faces had short hair and all the female faces had long hair. This explanation is consistent with existing 
research which suggests that sex discrimination of faces is indeed facilitated by the presence of a diagnostic hair length cue (Brown \& Perrett, 1993; Burton, Bruce \& Dench, 1993), which allows perceivers to engage in less cognitively taxing featural processing, as opposed to the configural processing that is thought to be necessary for the extraction of person information from faces (Bartlett \& Searcy, 1994; Searcy \& Bartlett, 1996; Macrae \& Martin, 2007; Martin \& Macrae, 2007; Mason \& Macrae, 2004). The results of Cloutier and colleagues strongly suggest that it is easier to extract group information from faces than person information, and further that this ease of extraction is both a fundamental property of group information, and a result of the presence of a diagnostic cue for group membership, which is absent for individual identification.

\subsection{The Present Research}

Despite this positive evidence in favor of the easier extraction of group information than person information from faces, we argue that the question remains unsettled. First, as noted, ERP evidence seems to favor equally simple and equally quick extraction of group and person information (Bentin \& Deouell, 2000). Second, and more fundamentally, research on group and person processes in impression formation has challenged one of the fundamental premises of the continuum model, through which Cloutier and colleagues interpret their results - namely the claim that processing group information is a simpler mental operation in general than processing person information (Skorich \& Mavor, 2013; see also Monroe et al., 2018). In this research, Skorich and Mavor (2013) deconstructed studies typically invoked as providing strong evidence for the processing advantage of group information over person information. In so doing, they showed that the processing advantage was not due to the relative ease of computing group information per se, but rather was a result of studies in this area having ubiquitously made group information co-vary with the simpler mental operation of memory-based processing (target characteristics already known), and person information 
having been made to co-vary with more complex data-driven processing (the target represents new information). Across three studies, Skorich and Mavor disentangled this covariation, and showed that group and person information was just as easy to decode once the group/person dimension was made orthogonal to the memory-based/data-driven dimension. This research, along with theorizing within the social identity/self-categorization tradition (Oakes, Haslam \& Turner, 1994; Turner, Hogg, Oakes, Reicher \& Wetherell, 1987; Turner, Oakes, Haslam \& McGarty, 1994), suggests that there is no a priori reason to postulate a processing advantage for group information over person information, in the way that the continuum model of impression formation does.

Given that Skorich and Mavor's (2013) findings challenge the notion that groupbased processing is inherently simpler than person-based processing, as assumed by the continuum model of impression formation, they cast doubt on Cloutier and colleagues' (2005, 2007 ) interpretation of their results. Specifically, in the absence of any a priori reason to expect group-based processing to be inherently and fundamentally simpler than person-based processing, it is necessary to explain the source of the difference that emerged between Cloutier and colleagues' sex group membership and individual identity tasks in a different way. A closer examination of Cloutier and colleagues' methodology provides hints as to possible sources of this difference: their group and person conditions differed not only in terms of the group versus person level of processing, but also in two additional respects more obviously related to processing speed. More specifically, the group conditions drew on a task in which (1) participants were asked to make a decision between only two "chunks" of information (i.e., the groups male and female), and (2) participants had the option of using a simple diagnostic cue to differentiate between the groups. The person conditions, on the other hand, were very different. "Familiar" and "unfamiliar" judgements cannot be made on the basis of any a priori diagnostic cue, but rather require some form of search from a large 
number of exemplars before a judgement of familiar/unfamiliar can be made. Therefore we argue that the person conditions involved a task in which (1) participants were asked to make a decision involving multiple 'unchunked' pieces of information (i.e., searching an unbounded list of possible familiar persons), and (2) participants did not (and indeed, could not) have access to a simple diagnostic cue to make their decisions. The group and person conditions therefore differ not only in terms of their level of representation (at either the group or person levels) but also in terms of the processing strategy (either a binary decision between two chunks or a decision between multiple unchunked entities) and in terms of participants' potential for reliance on the hair length cue. Each of these three co-occurring dimensions might reasonably have its own unique effect, in which case any one of them could be the source of the latency difference found by Cloutier and colleagues.

It must be emphasized that the covarying dimensions we discuss here were not introduced by Cloutier and colleagues, but were rather the result of these researchers adapting experimental conditions commonly employed in the face-processing literature to address different questions (e.g., Bentin \& Deouell, 2000; Bruce, 1983; Bruce et al., 1987; Eimer, 2000b; Freeman et al., 2010; O’Toole, Abdi, Deffenbacher \& Valentin, 1993; Sergent, 1986) and, more significantly, from adopting an understanding of group and person representations from the broader literature on stereotyping and person perception (Bodenhausen, Macrae \& Sherman, 1999; Fiske \& Taylor, 1991; Hamilton \& Sherman, 1996; Sherman \& Frost, 2000; Sherman, Macrae \& Bodenhausen, 2000). In this literature, group-level processing is commonly treated as involving some degree of simplification, in which group members are represented as interchangeable parts of a global, heuristic whole. Processing groups, therefore, is thought to involve a 'chunked' processing strategy (Sherman et al., 2000), in which the complexity of representing each group member is overridden by the less cognitively taxing process of employing pre-formed chunks of information (see Broadbent, 
1975 and Miller, 1956, for discussions of the efficiency of chunked processing). In direct contrast to this understanding of groups, person-level processing is commonly treated as involving some degree of detail-focus, in which each person is represented as a unique entity relative to each other person. Processing persons, therefore, is thought to involve an 'unchunked' processing strategy, in which the simplified chunks of information (the social group to which the persons belong) are downplayed in favor of the more resource-intensive process of employing distinct representations for each person simultaneously.

These understandings of groups and persons from the broader literature on stereotyping and person perception are problematic because they assume that the group versus person dimension naturally co-occurs with the 'chunked' versus 'unchunked' processing strategy dimension, when in fact these dimensions can be straightforwardly disentangled. Groups can certainly be processed as separate and distinct entities simultaneously — employing an 'unchunked' processing strategy — for example, when a census taker wishes to gauge the number of different ethnic groups in their community. Similarly, persons can undoubtedly be processed as simple chunks of information, made up of lower level interchangeable parts — employing a 'chunked' processing strategy — for example, when an investigator wishes to classify security camera footage to track the progress of a specific offender before and after a criminal event, or when a parent wishes to sort their photos according to which of their two children is represented. By disentangling the group/person dimension from the chunked/unchunked processing dimension, it then becomes possible to examine whether it is indeed the group versus person dimension that leads to the processing advantage seen in Cloutier and colleagues' $(2005,2007)$ studies as they argue, or whether it is instead the co-varying dimension of 'chunked' versus 'unchunked' processing. Disentangling the group/person dimension from the chunked/unchunked dimension also allows the presence versus absence of the diagnostic cue to be disentangled from the 
group/person dimension. In unchunked circumstances, it is logically impossible to have a single cue that will allow a perceiver to distinguish between all the entities with which they are being presented. In chunked tasks, however, in which a binary decision is being made, a single cue can be made to be diagnostic of the required decision. In Cloutier and colleagues' $(2005,2007)$ studies, therefore, it was only possible to have a hair length cue in their group tasks, because only these were binary, chunked tasks. When the group/person dimension is made orthogonal to the chunked/unchunked dimension, however, it becomes possible to introduce a single hair length cue that is diagnostic of personal identity — in particular, in a person-level binary chunked task, similar to Cloutier and colleagues' sex group membership task, but at the person-level. In this way, it also becomes possible to examine whether it is the group/person dimension per se that is the source of the processing advantage seen in Cloutier and colleagues' studies, or, rather, whether it is the presence of a diagnostic cue.

To summarize, then, the main aims of the current paper are (1) to explore whether group information or person information is easier to extract from faces or whether they are equally easy to extract; and (2) to determine whether the processing advantage attributed to group over person information in prior research in fact emerges from chunked versus unchunked processing, and from the presence versus absence of a diagnostic cue, with which this information has been made to co-vary. We present four experiments assessing these questions. In Experiment 1 and 2, we compare the extraction of group versus person information under chunked and unchunked processing strategy conditions. In Experiment 2, we also explore familiarity with the groups and persons as another possible source of any latency differences. In the third experiment, we assess the impact of the presence versus absence of a diagnostic cue on group and person face processing. Finally, in Experiment 4, we introduce and test the idea that prototypicality of face exemplars is a more important 
determinant of ease and speed of extracting information from faces than the group or personlevel nature of the task.

Given the analysis provided above, we make the general hypothesis that there will be no overall difference in the extraction of group versus person information from faces once the group/person dimension is disentangled from those dimensions with which it has been made to co-vary in prior research. We make the more specific predictions that a latency advantage will be observed: in chunked conditions relative to unchunked conditions; when a diagnostic cue is present relative to when a diagnostic cue is absent; when the groups or persons are more familiar to participants relative to when they are less familiar; and when the presented face instances are more prototypical of the group or person of which they are exemplars. Finding support for these hypotheses would be to corroborate, but add to, Bruce and Young's (1986) face recognition model and to challenge the group versus person distinction underlying the continuum model of impression formation (Fiske \& Neuberg, 1990).

\section{Experiment 1}

In this first Experiment, we attempt to disentangle the group/person dimension from the chunked/unchunked processing dimension, in order to assess which is the source of the latency advantage observed in prior research. Specifically, we adopt Cloutier and colleagues' $(2005 ; 2007)$ sex group membership and person familiarity conditions virtually unchanged as our 'chunked group' and 'unchunked person' tasks, respectively. We remove the diagnostic hair length cue from the chunked group task, however, because we assess the independent effect of diagnostic cue separately in Experiment 3. In addition to these adapted tasks, we introduce a novel 'chunked person' task. Given that a chunked task is one in which participants are asked to categorize face exemplars as members of one of two possible chunks (e.g., male versus female in Cloutier and colleagues' sex group membership and our 'chunked group' task), we ask participants in our chunked person task to decide whether each 
of a number of photos represents one person or another person. In particular, we ask participants to decide whether each of the individual face stimuli is Johnny Depp or Brad Pitt. In this way, we are able to assess whether any latency advantages are due to the group versus person distinction - by comparing the chunked group task to the chunked person task - or to the chunked versus unchunked processing strategy dimension — by comparing the chunked person task and the chunked group task to the unchunked person task.

Consistent with the analysis provided above, we hypothesize that:

H1. In spite of sufficient power (as determined by an a priori power analysis and equivalence test presented in the results section), there will be no difference between the chunked group task and the chunked person task, because each of these tasks invoke the same (relatively simple) processing strategy;

H2. The chunked group task will show faster reaction times than the unchunked person task, because the chunked group task invokes the simpler chunked processing strategy; and

H3. The chunked person task will show faster reactions times than the unchunked person task, because the chunked person task invokes the simpler chunked processing strategy.

\subsection{Method}

\subsubsection{Participants}

Twenty-four participants were recruited from the general population and from a pool of undergraduate psychology students. Sixteen participants were female and eight were male. The mean age was 20.96 years, with a standard deviation of 3.12 .

\subsubsection{Design}

The experiment employed a 3 condition (chunked group/chunked person/unchunked person) within-participants factorial design. The dependent variable was response latency. 


\subsubsection{Procedure}

The entire experiment was conducted on Windows computers in a computer laboratory. Millisecond Inquisit v. 2 was used to present all stimuli and collect all data. The stimuli consisted of 80 photos of various faces. Each photo was grayscale with a white background and standardized in size to $10 \mathrm{~cm} \times 10 \mathrm{~cm}$. The faces all displayed a neutral expression and were all directed forward.

The experiment consisted of three blocks. In each block participants were asked to press one of two keys to indicate whether a face was, depending on the block: male or female, a familiar or unfamiliar person, Johnny Depp or Brad Pitt. The relevant labels were displayed on screen throughout the block, on the side of the screen that corresponded to the associated key press. The response keys used for the reaction time task were the ' $\mathrm{g}$ ' key and the ' $k$ ' key. The meaning of these keys was counterbalanced across participants. Each trial consisted of the appearance of a fixation cross for $1000 \mathrm{~ms}$, followed by a face that remained on screen until participants made a response. Participants were asked to be as quick and as accurate as possible for each trial, as their response and reaction times were recorded ${ }^{1}$. Each experimental block was preceded by a practice block, identical in procedure to the experimental block, but with fewer (eight) photos presented. The experimental blocks, the order of which was counterbalanced across participants, were constructed as follows:

2.1.3.1 Chunked group block: This block was equivalent to the unrotated 'sex' trials of Cloutier and Macrae (2007) and the unblurred 'sex' trials of Cloutier et al. (2005), but with hair-length controlled. Participants were presented with the faces of 20 unfamiliar people, in random order. Of those 20 faces, 10 were male and 10 were female. Five males and five females had short hair, and five males and five females had long hair. Participants were asked

\footnotetext{
${ }^{1}$ To avoid repetition in subsequent Method sections, note that response keys, their counterbalancing,
} and the time-course of individual trials were as described here across all Experiments reported in this paper. 
to categorize each face as quickly and as accurately as possible, as either male or female. The screen labels were 'male' and 'female'.

2.1.3.2 Unchunked person block: This block was equivalent to the unrotated 'identity' trials of Cloutier and Macrae (2007) and the unblurred 'identity' trials of Cloutier et al. (2005). Participants were presented with the faces of 20 people, in random order; 10 were celebrities that were well-known to young Australians (e.g., Paris Hilton, Britney Spears, Leonardo di Caprio, Orlando Bloom) and 10 were unfamiliar. Five celebrities and five strangers each were male and female. Participants were asked to decide as quickly and as accurately as possible whether each face was either familiar or unfamiliar. The screen labels were 'familiar' and 'unfamiliar'.

2.1.3.3 Chunked person block: In this block, participants were presented with 20 different photos of either Johnny Depp or Brad Pitt, in random order. Of the 20 photos, 10 were of Johnny Depp and 10 were of Brad Pitt. Photos were drawn from a range of situations, time periods and events (see Figure 1). Participants were asked to categorize each photo as quickly and as accurately as possible, as either Johnny Depp or Brad Pitt. The screen labels were 'Depp' and 'Pitt'.

Following the three blocks, participants were asked to indicate their age, their gender, their ethnic background, and whether English was their first language. Participants were then thanked for their time and farewelled.

\subsection{Results}

\subsubsection{Power Analysis}

Given that $\mathrm{H} 1$ predicts a null effect, a power analysis was conducted based on the group vs. person response latency effects in Cloutier et al. (2005) and Cloutier and Macrae's (2007) experiments. Effect sizes ranged from a maximum of $d=3.63$ in Cloutier et al.'s (2005) first experiment to a minimum of $d=2.22$ in Cloutier et al.'s third experiment. Non- 
central 95\% and 99\% confidence intervals (Smithson, 2002) were constructed around this latter (smallest) value. These revealed lower bound estimates for the group vs. person effect of $d=1.25$ and $d=0.98$, respectively. In the interest of being conservative, therefore, an effect of $d=0.98$ - that for the $99 \%$ confidence interval - was deemed the smallest meaningful effect for an experiment of this sort. Deriving this value allowed us to determine whether there would be sufficient power in the current experiments to detect an effect of $d=$ 0.98 . With a sample size of 24 and an alpha-level of .05 , power is equal to .83 , which is above the value of .8, considered statistically powerful using Cohen's $(1988,1992)$ criteria.

\subsubsection{Planned Comparisons}

For each participant, median reaction times were calculated for each condition. Trials on which errors were made ( $8.3 \%$ of trials) were excluded from all analyses. The reaction time data thus obtained (see Figure 2) were submitted to three planned comparisons:

2.2.2.1 Sex and identity replication. First, the chunked group block was compared to the unchunked person block. Participants were significantly faster at responding to indicate that a face was male or female than they were at responding to indicate that a face was a familiar or unfamiliar person, $t(23)=-7.83, p<.001, d=1.59, \eta^{2}=.727$.

2.2.2.2 Chunked processing conditions. The chunked person block was compared to the chunked group block. This revealed no significant difference between these blocks, $t$ (23) $=-1.088, p=.288, d=.22, \eta^{2}=.049$.

2.2.2.3 Person-level conditions. The chunked person block was compared to the unchunked person block. Participants were significantly faster at responding to indicate that a face was Johnny Depp or Brad Pitt than they were at responding to indicate that a face was a familiar or unfamiliar person, $t(23)=-8.652, p<.001, d=1.77, \eta^{2}=.765$. 


\subsection{Discussion}

As hypothesized, no difference was found between response times for the chunked group task and the chunked person task (in line with H1). However, responses to the chunked group task were found to be faster than those to the unchunked person task (supporting H2). Similarly, responses to the chunked person task were also found to be faster than those to the unchunked person task (supporting H3). Together, then, these results suggest that there is no obvious processing advantage for group over person information per se. Rather, it appears that chunked processing is easier and faster than unchunked processing, irrespective of whether it is employed at the group or person levels.

\section{Experiment 2}

In Experiment 2, we build on the results of Experiment 1 by again manipulating group versus person processing orthogonally to a manipulation of the degree of chunked versus unchunked processing. In the original fully unchunked tasks, we do not know how many possible targets a participant might consider when making their familiar/unfamiliar choice, and therefore this is harder to control. To examine this process in a more systematic way, therefore, we explicitly manipulate the number of chunks participants are asked to process simultaneously, with the goal of assessing the speed of making yes/no decisions about a single group or person chunk relative to two group or person chunks, or four group or person chunks simultaneously. We also measure participants' familiarity with the group and person chunks, as familiarity is known to be a key facilitator of the speed of processing for individual identity (Quinn, Mason \& Macrae, 2009). We build on this idea by assuming that the same familiarity advantage should also accrue for group decisions. As such, we posit that any difference in processing speed between conditions should disappear when relative familiarity with the groups and persons is controlled for. This would indicate that familiarity 
is a secondary source of any processing advantage beyond the chunked/unchunked processing strategy, but, again, one that affects groups and persons in similar ways. For Experiment 2, we hypothesize that:

H1. In spite of sufficient power (as determined by the power analysis reported in the results section of Experiment 1), there will be no overall difference in response time between the group and person tasks, particularly when relative familiarity is controlled for.

$\mathrm{H} 2$ : The one-chunk conditions will show faster response times than the two-chunk conditions, for both the group and person tasks.

H3: The two-chunk conditions will show faster responses times than the four-chunk conditions, for both the group and person tasks.

\subsection{Method}

\subsubsection{Participants}

Twenty-four participants were recruited from a pool of first-year undergraduate psychology students. Thirteen participants were female and eleven were male. The mean age was 20.42 years, with a standard deviation of 2.62 .

\subsubsection{Design}

The experiment employed a 2 (type of information: person/group) $\times 3$ (number of chunks: one/two/four) within-participants factorial design. The dependent variable was response latency.

\subsubsection{Procedure}

The entire experiment was conducted on Windows computers in a computer laboratory. Millisecond Inquisit v. 3 was used to present all stimuli and collect all data. The stimuli consisted of 240 photos of various faces. Each photo was grayscale with a white 
background and standardized in size to $10 \mathrm{~cm} \times 10 \mathrm{~cm}$. The faces all displayed a neutral expression and were all directed forward.

The experiment consisted of six experimental blocks, followed by a series of familiarity measures and demographic questions. In each of the experimental blocks, the task was to decide if a single presented face belongs to a set of one, two or four possible (person or group) targets, or not (see below for details). Prior to the experimental blocks, participants were asked to complete two practice blocks to familiarize themselves with the procedure of the subsequent experimental blocks. The practice blocks consisted of an old/young task - in which participants were asked to indicate if each of 24 faces was old or young — and a Sean Connery/Clint Eastwood task - in which participants were asked to indicate if each of 24 faces was Sean Connery or Clint Eastwood. The experimental blocks (the order of which was counterbalanced across participants) were constructed as follows:

3.1.3.1 One-chunk person block: In this block, the task was to decide if a single presented face belongs to a single identified person target, or not. Participants were presented with 24 different photos of two different celebrities, in random order. Of the 24 photos, 12 were of one celebrity (target) and 12 were of the other (distractor) celebrity. We also used two different sets of celebrity pairs to increase generality, but also to allow for more variation in the familiarity measure taken later.

Depending on the counterbalanced condition to which they were randomly assigned, participants were presented either with target photos of Johnny Depp or Brad Pitt, or with distractor photos of Tom Cruise or Hugh Jackman. Photos were drawn from a range of situations, time periods and events, and were different from the photos of the same celebrities included in the other blocks. Depending on counterbalanced condition, participants were asked to respond to the questions "Is this Johnny Depp?" or “Is this Tom Cruise?" for each photo as quickly and as accurately as possible. Participants were asked to press the ' $\mathrm{g}$ ' key if 
the face belonged to [Johnny Depp/Tom Cruise] and to press the ' $\mathrm{k}$ ' key if the face did not belong to [Johnny Depp/Tom Cruise]. The screen labels were 'Yes' and 'No', respectively.

3.1.3.2 Two-chunk person block: In this block, the task was to decide if a single presented face belongs to either of two identified person targets, or not. Participants were presented with 24 different photos of four celebrities, in random order. Of the 24 photos, 6 were of each of the four celebrities, with two of the celebrities being potential targets and two being distractors. Depending on the counterbalanced condition to which they were randomly assigned, participants were presented either with (1) target photos of Johnny Depp or Ryan Gosling, or distractor photos of Brad Pitt or Leonardo Di Caprio, or (2) with target photos of Tom Cruise or Robert Pattinson, or distractor photos of Hugh Jackman or Ashton Kutcher. Photos were drawn from a range of situations, time periods and events, and were different from the photos of the same celebrities included in the other blocks. Participants were asked to respond to the question "Is this Johnny Depp or Ryan Gosling" or "Is this Tom Cruise or Robert Pattinson?" for each photo as quickly and as accurately as possible. Participants were asked to press the ' $\mathrm{g}$ ' key if the face belonged to [Johnny Depp or Ryan Gosling/Tom Cruise or Robert Pattinson], and to press the ' $\mathrm{k}$ ' key if the face did not belong to [Johnny Depp or Ryan Gosling/Tom Cruise or Robert Pattinson]. The screen labels were 'Yes' and 'No', respectively.

3.1.3.3 Four-chunk person block: In this block, the task was to decide if a single presented face belongs to one of four identified person targets, or not. Participants were presented with 24 different photos of eight celebrities, in random order. Of the 24 photos, three were of each of Johnny Depp, Ryan Gosling, Tom Cruise, Robert Pattinson (possible targets), Brad Pitt, Hugh Jackman, Leonardo Di Caprio, and Ashton Kutcher (possible distractors). Photos were drawn from a range of situations, time periods and events, and were different from the photos of the same celebrities included in the other blocks. Participants 
were asked to respond to the question "Is this Johnny Depp, Ryan Gosling, Tom Cruise or Robert Pattinson?" for each photo as quickly and as accurately as possible. Participants were asked to press the ' $\mathrm{g}$ ' key if the face belonged to Johnny Depp, Ryan Gosling, Tom Cruise or Robert Pattinson, and to press the ' $\mathrm{k}$ ' key if the face did not belong to Johnny Depp, Ryan Gosling, Tom Cruise or Robert Pattinson. The screen labels were 'Yes' and 'No', respectively.

3.1.3.4 One-chunk group block: In this block, the task was to decide if a single presented face belongs to a single identified group target, or not. Participants were presented with 24 photos of unknown people of two different ethnic backgrounds, in random order. Of the 24 photos, 12 were of people of one ethnic background and 12 were of people of the other ethnic background. Depending on the counterbalanced condition to which they were randomly assigned, participants were presented either with photos of people of Caucasian and Asian background, or with photos of people of Indian and African background. All the photos included in this block were different from the photos included in the other blocks. Participants were asked to respond to the question "Is this person Caucasian?" or "Is this person Indian?" for each photo as quickly and as accurately as possible. They were asked to press the ' $\mathrm{g}$ ' key if the face belonged to a person of [Caucasian/Indian] background, and to press the ' $\mathrm{k}$ ' key if the face did not belong to a person of [Caucasian/Indian] background. The screen labels were 'Yes' and 'No', respectively.

3.1.3.5 Two-chunk group block: In this block, the task was to decide if a single presented face belongs to either of two identified group targets, or not. Participants were presented with 24 photos of unknown people of four ethnic backgrounds, in random order. Of the 24 photos, 6 were of people of each of the four ethnic backgrounds. Depending on the counterbalanced condition to which they were randomly assigned, participants were presented either with photos of people of Caucasian or Indigenous Australian (targets), or 
Asian or Papuan background (distractors), or with photos of people of Indian or Middle Eastern (targets), or African or Polynesian (distractors) background (see Figure 3). All the photos included in this block were different from the photos included in the other blocks. Participants were asked to respond to the question "Is this person Caucasian or Indigenous Australian?" or "Is this person Indian or Middle Eastern?" for each photo as quickly and as accurately as possible. Participants were asked to press the ' $g$ ' key if the face belonged to a person of [Caucasian or Indigenous Australian/Indian or Middle Eastern] background, and to press the ' $\mathrm{k}$ ' key if the face did not belong to a person of [Caucasian or Indigenous Australian/Indian or Middle Eastern] background. The screen labels were 'Yes' and 'No', respectively.

3.1.3.6 Four-chunk group block: In this block, the task was to decide if a single presented face belongs to one of four identified group targets, or not. Participants were presented with 24 photos of unknown people of eight ethnic backgrounds, in random order. Of the 24 photos, three were of people of each of the following ethnic backgrounds: Caucasian, Indigenous Australian, Indian, Middle Eastern (targets), or Asian, African, Papuan and Polynesian (distractors). All the photos included in this block were different from the photos included in the other blocks. Participants were asked to respond to the question "Is this person Caucasian, Indigenous Australian, Indian or Middle Eastern?” for each photo as quickly and as accurately as possible. Participants were asked to press the ' $\mathrm{g}$ ' key if the face belonged to a person of Caucasian, Indigenous Australian, Indian, or Middle Eastern background, and to press the ' $\mathrm{k}$ ' key if the face did not belong to a person of Caucasian, Indigenous Australian, Indian, or Middle Eastern background. The screen labels were 'Yes' and 'No', respectively.

Following the presentation of all six experimental blocks (counterbalanced across participants), participants were asked to rate their familiarity with each of the celebrities from 
the previous tasks, and to estimate the number of people of each ethnic background they knew well. The person familiarity ratings consisted of a response to the question "How familiar would you say you are with [celebrity's name]?” for each of the celebrities, on a 7point scale from (1) not at all familiar to (7) very familiar. The group familiarity ratings, derived from Linville, Fischer and Yoon (1996), consisted of an open-ended estimate in response to the question "roughly how many people of [ethnicity label] background would you consider close friends of yours?" for each of the ethnic backgrounds.

Following these familiarity ratings, participants were asked to indicate their age, their gender, their ethnic background, and whether English was their first language. Participants were then thanked for their time and farewelled.

\subsection{Results}

\subsubsection{Overall Analysis}

For each participant, median reaction times were calculated for each condition. Trials on which errors were made ( $10.5 \%$ of trials) were excluded from all analyses. The reaction time data thus obtained were submitted to a 2 (type of information: person/group) $\times 3$ (number of chunks: one/two/four) within-participants factorial ANOVA. This revealed a significant effect of type of information, $F(1,23)=14.592, p=.001$, a significant effect of number of chunks, $F(1,22)=35.06, p<.001$ and a significant interaction, $F(1,21)=7.965$, $p=.002$. The positive increase from the one-chunk condition, through the two-chunk condition to the four-chunk condition for the number of chunks factor was shown to be linear, $F(1,23)=72.643, p<.0001$.

\subsubsection{Relative Familiarity Index}

Given the known impact of familiarity on face perception, participants' familiarity with the person targets was compared to their familiarity with the group targets. First, each of the group familiarity measures was (1) screened for outliers, which were replaced with the 
next highest values; (2) normalized using a logarithmic transformation, and (3) standardized onto a seven-point scale. A paired-samples $t$-test was then conducted to compare the mean of these standardized group familiarity measures with the mean of the person familiarity measures. This revealed that participants were significantly more familiar with the person targets $(M=4.53)$ than with the group targets $(M=2.84), t(23)=4.495, p<.0001$. Given this difference in familiarity between person and group targets, a relative familiarity index was created. The mean of the standardized group familiarity measures was subtracted from the mean of the person familiarity measures to create this measure of relative familiarity, such that a negative score suggested more familiarity with the groups than the persons, whereas a positive score suggested more familiarity with the persons than the groups. The relative familiarity measure was entered as a covariate in the $2 \times 3$ within-participants factorial ANOVA. This revealed that the previously significant effect of type of information (person/group) had disappeared, $F(1,23)=2.493, p=.129$, as had the significant interaction, $F(1,21)=1.997, p=.161$. In contrast, the significant effect of number of chunks remained highly significant after controlling for relative familiarity, $F(1,22)=13.546, p<.001$ (see Figure 4, where Means are adjusted for the level of relative familiarity).

\subsubsection{Planned Comparisons}

3.2.3.1 One-chunk conditions. The single chunk person condition was compared to the single chunk group condition. This revealed no significant difference between these conditions, $t(23)=-1, p=.328$.

3.2.3.2 Two-chunk conditions. The two-chunk person condition was compared to the two-chunk group condition. This revealed a significant difference between these conditions, $t$ $(23)=-3.036, p=.006$, such that participants were faster at responding on the two-chunk person task $(M=713.56 \mathrm{~ms}, S D=130.85)$ than on the two-chunk group task $(M=847.94 \mathrm{~ms}$, $S D=277.53)$ 
In order to assess whether relative familiarity was partly responsible for this difference, a two-chunk relative familiarity index was created. The mean of the two standardized group familiarity measures relevant to each participant's counterbalanced condition was subtracted from the mean of the two-person familiarity measures relevant to each participant's counterbalanced condition. The two-chunk relative familiarity index thus created was then entered as a covariate in a one-way ANOVA. This revealed that the previously significant difference between the person and group tasks had disappeared, $F$ (1, 22) $=.396, p=.536$.

3.2.3.3 Four-chunk conditions. The four-chunk person condition was compared to the four-chunk group condition. This revealed a significant difference between these conditions, $t(23)=-3.522, p=.002$, such that participants were faster at the four-chunk person task $(M=892.69 \mathrm{~ms}, S D=216.61)$ than the four-chunk group task $(M=1137.85 \mathrm{~ms}$, $S D=396.50$ ). To assess the effect of relative familiarity on the four-chunk conditions, the overall relative familiarity index was entered as a covariate in a one-way ANOVA. This revealed that the previously significant difference between the person and group tasks had become non-significant, $F(1,22)=3.503, p=.075$.

3.2.3.4 Person conditions. The one-chunk person condition was compared to the two-chunk person condition. This revealed a significant different between these conditions, $t$ $(23)=-3.021, p=.006$, such that participants were faster at the one-chunk person task $(M=$ $658.88 \mathrm{~ms}, S D=113.82)$ than the two-chunk person task $(M=713.56 \mathrm{~ms}, S D=130.85)$. The two-chunk person condition was then compared to the four-chunk person condition. This revealed a significant difference between these conditions, $t(23)=-6.546, \mathrm{p}<.0001$, such that participants were faster at the two-chunk person task $(M=713.56 \mathrm{~ms}, S D=130.85)$ than the four-chunk person task $(M=892.69 \mathrm{~ms}, S D=216.61)$. 
3.2.3.5 Group conditions. The one-chunk group condition was compared to the twochunk group condition. This revealed a significant different between these conditions, $t(23)$ $=-4.179, p<.0001$, such that participants were faster at the one-chunk group task $(M=$ $684.54 \mathrm{~ms}, S D=155.98)$ than the two-chunk group task $(M=847.94 \mathrm{~ms}, S D=277.53)$. The two-chunk group condition was then compared to the four-chunk group condition. This revealed a significant difference between these conditions, $t(23)=-4.273, p<.0001$, such that participants were faster at the two-chunk group task $(M=847.94 \mathrm{~ms}, S D=277.53)$ than the four-chunk group task $(M=1137.85 \mathrm{~ms}, S D=396.50)$.

\subsection{Discussion}

As hypothesized, we found no overall difference between the group and person tasks when relative familiarity was controlled for, across each of the number of chunks conditions (in line with H1). We also found that participants were faster in the one-chunk conditions than in the two-chunk conditions for both group and person tasks (supporting H2), and that they were faster in the two-chunk conditions than the four-chunk conditions for both group and person tasks (H3). We found that participants were more familiar overall with the person targets than the group targets, and that this explained the baseline differences in response times found in favor of the person tasks. Together, then, these results build on those of Experiment 1 in again suggesting that there is no processing advantage for either group or person information per se. Instead, it appears that participants' response speed is determined by the number of chunks that they are asked to process simultaneously and their familiarity with the specific persons and groups.

\section{Experiment 3}

In Experiment 3, we examine the claim that the presence of a diagnostic cue facilitates the identification of group membership in a face. Consistent with our analysis presented earlier, however, we suggest that a diagnostic cue need not only facilitate group membership 
identification, but should also facilitate individual identification when the person decision is a binary one. We adopt a similar methodology to that of Experiment 1 . Specifically, we replicate the chunked group task and the chunked person task from that experiment exactly in the cue-absent group and cue-absent person conditions of this experiment, respectively. We also modify the chunked group task and chunked person task from Experiment 1, such that a hair length cue is made to be diagnostic of sex group membership and individual identity in the cue-present group and cue-present person conditions of this experiment, respectively. Specifically, in the cue-present group task, all the male faces are made to have short hair, while all the female faces are made to have long hair. In the cue-present person task, Johnny Depp and Brad Pitt are replaced with George Clooney and Bruce Willis, such that hair length is made to co-vary with personal identity; that is, George Clooney has hair in all photos and Bruce Willis is bald in all photos.

For Experiment 3, we hypothesize that:

H1. In spite of sufficient power (as determined by the power analysis reported in the results section of Experiment 1), there will be no difference in response times between the group and person tasks overall.

H2. The cue-present conditions will show faster response times than the cue-absent conditions, irrespective of the group versus person level of the task.

\subsection{Method}

\subsubsection{Participants}

Twenty-eight participants were recruited from a pool of first-year undergraduate psychology students. Four participants were excluded from the experiment because they indicated to the experimenter that they were unfamiliar with one or more of the celebrities presented in the study. Twenty-four participants therefore completed the experiment. Twenty 
participants were female and four were male. The mean age was 18.79 years, with a standard deviation of 1.22 .

\subsubsection{Design}

The experiment employed a 2 (type of information: person/group) $\times 2$ (cue availability: cue-absent/cue-present) within-participants factorial design. The dependent variable was response latency.

\subsubsection{Procedure}

The entire experiment was conducted on Windows computers in a computer laboratory. Millisecond Inquisit v. 3 was used to present all stimuli and collect all data. The stimuli consisted of 80 photos of various faces. Each photo was grayscale with a white background and standardized in size to $10 \mathrm{~cm} \times 10 \mathrm{~cm}$. The faces all displayed a neutral expression and were all directed forward.

The experiment consisted of four blocks. In each block participants were asked to press one of two keys to indicate whether a face was, depending on the block, male or female, George Clooney or Bruce Willis, or Johnny Depp or Brad Pitt (see below for details). Each experimental block was preceded by a practice block, identical in procedure to the experimental block, but with fewer (eight) photos presented. The experimental blocks, the order of which was counterbalanced using a $4^{\text {th }}$ order Latin square (Alimena, 1962), were constructed as follows:

4.1.3.1 Group (cue-present) block: This block was based on the chunked group block from Experiment 1, but hair length was made to be diagnostic of category membership such that all males had short hair, and all females had long hair. It was thus possible for participants to engage in featural processing.

4.1.3.2 Group (cue-absent) block: This block was identical to the chunked group block from Experiment 1. 
4.1.3.3 Person (cue-present) block: This block was identical to the chunked person block from Experiment 1, except that the photos presented were of George Clooney and Bruce Willis, rather than Johnny Depp and Brad Pitt. Importantly, this difference meant that hair length was diagnostic of identity, such that George Clooney had hair in all the photos, while Bruce Willis was bald in all the photos (see Figure 5). Participants could therefore use hair length as a cue to make their person-level identification decisions.

4.1.3.4 Person (cue-absent) block: This block was identical to the chunked person block from Experiment 1.

Following all four blocks, participants were asked to indicate their age, their gender, their ethnic background, and whether English was their first language. Participants were then thanked for their time and farewelled.

\subsection{Results}

\subsubsection{Overall Analysis}

For each participant, median reaction times were calculated for each condition. Trials on which errors were made $(6.5 \%$ of trials) were excluded from all analyses. The reaction time data thus obtained were submitted to a 2 (type of information: person/group) $\times 2$ (cue availability: cue-present/cue-absent) within-participants factorial ANOVA. This revealed a significant effect of cue availability, $F(1,23)=29.013, p<.001-$ such that participants were faster when a cue was present than when it was absent - no effect of type of information (person/group), $F(1,23)=.021, p=.887$, and no interaction, $F(1,23)=1.633, p=.214($ see Figure 6).

\subsection{Discussion}

As hypothesized, we found no overall difference between group and person tasks (in line with H1). We did, however, uncover a response advantage for cue-present conditions over cue-absent conditions, which was not moderated by the type of information. Consistent 
with the findings of Experiments 1 and 2, these results suggest that group and person information is equally easy to extract from faces. Furthermore, supporting H2, the presence versus absence of a diagnostic cue was an important determinant of processing speed, and, importantly, this processing advantage was afforded to both group and person processing equally. Together, then, Experiments 1, 2, and 3, all suggest that group and person information is equivalent in its task demands, and that the secondary factors of chunking, familiarity, and the presence of a diagnostic cue are the key determinants of processing speed in the extraction of both group and person information from faces.

\section{Experiment 4}

In our final experiment, we aimed to remove a possible confound from Experiments 1 to 3 , and also to go beyond examining previously identified determinants of the speed of extracting group and person information from faces. In Experiments 1, 2, and 3, participants were asked to respond to different sets of stimuli in each condition. This leaves open the possibility that any differences observed between those conditions were due to these different stimuli, rather than to the factors we aimed to manipulate. To correct for this, in the current experiment participants were presented with the same set of stimuli across all conditions, the only difference between conditions therefore being the decision — at the person-level or at the group-level - participants were asked to make. In order to do this, the group task was made to be an age-group (old versus young) task, as this allowed us to use the same individuals across group conditions. Specifically, the stimuli were photos of Clint Eastwood and Sean Connery — which represents the dichotomous decision participants were asked to make in the person conditions - when they were young and old — which represents the dichotomous decision participants were asked to make in the group conditions.

In going beyond the previously identified factors of chunking, familiarity and the presence of diagnostic cues, we identified the prototypicality of face exemplars as a possible 
additional determinant of processing speed. We define prototypicality as the degree to which an exemplar represents the central tendency of the entity of which it is a part, in contrast to an entity of which it is not a part (McGarty, 1999; Rosch, 1978). For age groups, prototypicality is therefore instantiated as the degree to which a specific photo captures the characteristics that define what it is to be young relative to the characteristics that define what it is to be old. For personal identity, prototypicality can be thought of as the degree to which a specific photo captures the characteristics that define what it is to be one person relative to the characteristics that define what it is to be a second person. Overall, the more prototypical an exemplar is of a particular group membership or personal identity, the faster a perceiver should be able to classify it as such.

Given the results of Experiments 1 to 3 and the analysis of prototypicality provided above, for Experiment 4, we hypothesize that:

H1: In spite of sufficient power (as determined by the power analysis reported in the results section of Experiment 1), there will be no difference in response times between group and person tasks overall.

H2: Greater prototypicality of face exemplars will be associated with faster response times relative to lower prototypicality of face exemplars, irrespective of the group or person nature of the task.

\subsection{Pilot Study}

In order to establish the person prototypicality of a number of photos of Clint Eastwood and Sean Connery, a pilot study was first conducted. Eighteen participants were asked to rate their agreement with the statement "In this photo, [Sean Connery/Clint Eastwood] looks recognizably and typically like [Sean Connery/Clint Eastwood]" on a 7point Likert scale from (1) strongly disagree to (7) strongly agree, for each of 20 photos of 
each celebrity under the age of 45 , and for each of 20 photos of each celebrity over the age of 50 (for a total of 80 photos).

In order to establish the group prototypicality of the same set of photos, participants were asked to rate their agreement with the statement "In this photo, this person looks stereotypically [young/old]" on a 7-point Likert scale, from (1) strongly disagree to (7) strongly agree for each photo.

The prototypicality data thus obtained was then sorted by photo, such that each photo had a rating both for its person prototypicality and its group prototypicality. The group prototypicality rating for each photo was then subtracted from the person prototypicality rating for that photo. The photos were then sorted by this derived difference score. The 24 photos (12 of Sean Connery, 12 of Clint Eastwood, half of each celebrity under the age of 45, and half of each celebrity over the age of 50) that were found to be most prototypical of the person depicted and LEAST prototypical of the age group depicted were deemed to be person prototypical. The 24 photos (12 of Sean Connery, 12 of Clint Eastwood, half of each celebrity under the age of 45 , and half of each celebrity over the age of 50) that were found to be most prototypical of the age group depicted and LEAST prototypical of the person depicted were deemed to be group prototypical. The 24 photos (12 of Sean Connery, 12 of Clint Eastwood, half of each celebrity under the age of 45 , and half of each celebrity over the age of 50) that were approximately equally prototypical of the person and the age group depicted were deemed to be neutral in their prototypicality.

In order to ensure that the three groups of photos were in fact different in terms of their prototypicality, three paired sample $t$-tests were conducted comparing the mean person prototypicality ratings to the mean group prototypicality ratings for each set of photos. As expected, the person prototypical photos were found to be significantly more prototypical of the person depicted $(M=5.24)$ than the group $(M=3.94), t(17)=7.058, p<.0001$. 
Similarly, the group prototypical photos were found to be significantly more prototypical of the age group depicted $(M=5.50)$ than the person $(M=4.43), t(17)=6.147, p<.0001$. The neutral prototypicality photos were found not to differ in terms of their person or group prototypicality ratings, $t(17)=-.399, p=.695$.

\subsection{Method}

\subsubsection{Participants}

Twenty-five participants were recruited from a pool of first-year undergraduate psychology students, and from the general population. One participant was excluded from the experiment because she indicated to the experimenter that she was unfamiliar with both Sean Connery and Clint Eastwood. Twenty-four participants therefore completed the experiment. Sixteen participants were female and eight were male. The mean age was 27.78 years, with a standard deviation of 6.84 .

\subsubsection{Design}

The experiment employed a 2 (Type of information: person/group) $\times 3$ (Prototypicality: person/neutral/group) within-participants factorial design. The dependent variable was response latency.

\subsubsection{Procedure}

The entire experiment was conducted on Windows computers in a computer laboratory. Millisecond Inquisit v. 3 was used to present all stimuli and collect all data. The stimuli consisted of 18 photos of Sean Connery under the age of 45, 18 photos of Sean Connery over the age of 50,18 photos of Clint Eastwood under the age of 45, and 18 photos of Clint Eastwood over the age of 50, for a total of 72 photos. Half of these 72 photos, therefore, depicted Sean Connery and half depicted Clint Eastwood, and, orthogonally, half the photos depicted an older person and half depicted a younger person (see Figure 7). As described in the pilot study section above, a third of these 72 photos were prototypical of the 
person depicted, a third were prototypical of the group depicted, and a third were equally prototypical of the person and the group. Each photo was grayscale with a white background and standardized in size to $10 \mathrm{~cm} \times 10 \mathrm{~cm}$. The faces all displayed a neutral expression and were all directed forward.

The experiment consisted of two experimental blocks, followed by a series of familiarity measures and demographic questions. In each of the experimental blocks, participants were asked to press one of two keys to indicate whether a face was, depending on the block: Sean Connery or Clint Eastwood, or an older or younger person (see below for details). The same 72 photos were used in both blocks. Prior to the experimental blocks, participants were asked to complete two practice blocks to familiarize themselves with the procedure of the subsequent experimental blocks. The practice blocks consisted of the group (cue-absent: male vs. female) and person (cue-absent: Johnny Depp vs. Brad Pitt) tasks described in Experiments 1 and 3. The experimental blocks, the order of which was counterbalanced across participants, were constructed as follows:

5.2.3.1 Person block: In this block, participants were presented with the 72 photos, in random order. Participants were asked to categorize each photo as quickly and as accurately as possible, as either Sean Connery or Clint Eastwood. The screen labels were 'Connery' and 'Eastwood'.

5.2.3.2 Group block: In this block, participants were presented with the 72 photos, in random order. Participants were asked to categorize each photo as quickly and as accurately as possible, as either an older person or a younger person. The screen labels were 'old' and 'young'.

Following the presentation of the two experimental blocks (counterbalanced across participants), participants were asked to rate their familiarity with Sean Connery and Clint Eastwood, and with people under the age of 45 and people over the age of 50 . The person 
familiarity ratings consisted of a response to the question "How familiar would you say you are with [Sean Connery/Clint Eastwood]?", on a 7-point scale from (1) not at all familiar to (7) very familiar. The group familiarity ratings consisted of a response to the question "How familiar would you say you are with [people under the age of 45/people over the age of 50] in general?", on a 7-point scale from (1) not at all familiar to (7) very familiar.

Following these familiarity ratings, participants were asked to indicate their age, their gender, their ethnic background, and whether English was their first language. Participants were then thanked for their time and farewelled.

\subsection{Results}

\subsubsection{Overall Analysis}

For each participant, median reaction times were calculated for each condition. Trials on which errors were made ( $10.8 \%$ of trials) were excluded from all analyses. The reaction time data thus obtained were submitted to a 2 (type of information: person/group) $\times 3$ (prototypicality: person/neutral/group) within-participants factorial ANOVA. This revealed no effect of type of information (person/group), $F(1,23)=.747, p=.396$, a significant effect of prototypicality, $F(1,22)=9.573, p=.001$, and a significant interaction, $F(1,22)=$ $25.504, p<.0001$ (see Figure 8).

\subsubsection{Planned Comparisons}

5.3.2.1 Person prototypicality conditions. The person prototypical person condition was compared to the person prototypical group condition. This revealed a significant difference between these conditions, $t(23)=-2.43, p=.023$, such that participants were faster at the person prototypical person task $(M=704.08 \mathrm{~ms}, S D=136.27)$ than the person prototypical group task $(M=781.19 \mathrm{~ms}, S D=149.47)$.

5.3.2.2 Group prototypicality conditions. The group prototypical person condition was compared to the group prototypical group condition. This revealed a significant 
difference between these conditions, $t(23)=3.098, p=.005$, such that participants were faster to respond on the group prototypical group task $(M=648.04 \mathrm{~ms}, S D=99.92)$ than on the group prototypical person task $(M=758.71 \mathrm{~ms}, S D=155.89)$.

5.3.2.3 Neutral prototypicality conditions. The neutral prototypical person condition was compared to the neutral prototypical group condition. This revealed no significant difference between these conditions, $t(23)=1.497, p=.148$.

\subsection{Discussion}

As predicted, there was no overall difference in response times between the group and person tasks (in line with $\mathrm{H} 1$ ). Supporting H2, however, there was a processing speed advantage for the high prototypicality conditions relative to the neutral and low prototypicality conditions for both the group and person tasks. These results suggest again that there is no processing advantage for group over person information. These results also go beyond previous findings in showing the importance of prototypicality in the speed of extracting group and person information from faces. In particular, face exemplars that are group prototypical will show a group processing speed advantage, while face exemplars that are person prototypical will show a person processing speed advantage. As such, these results provide some preliminary insights into the conditions under which perceivers will be more likely to process at one level relative to another. We return to this point in the general discussion below.

\section{General Discussion}

In the current research, we aimed to explore the ease of extracting group and person information from faces, in order to test the conflicting predictions of Bruce and Young's (1986) face recognition model and Fiske and Neuberg's (1990) continuum model of impression formation. Across the four experiments, we found no evidence for the continuum model's suggestion that group information is processed more quickly, more easily, and earlier 
in the processing stream than person information. Instead, we found that a processing advantage accrued for chunked over unchunked processing, for the presence versus absence of a diagnostic cue, for familiar over unfamiliar entities, and for prototypical over nonprototypical face exemplars - and, importantly, that each of these advantages accrued for both group and person tasks. Together, these results provide support for Bruce and Young's (1986) face recognition model and, specifically, for its contention that group membership information and personal identity information are both supported by similar processes, affording them the same processing advantages and disadvantages. These results also suggest that previous research found a processing advantage for group information over person information because group information was made to co-vary ubiquitously, and unnecessarily, with several other processes. We show however, that once these confounds are eliminated, group information has no processing advantage. These findings have important implications for Bruce and Young's (1986) face recognition model, for the continuum model of impression formation (Fiske \& Neuberg, 1990), and they also provide a novel avenue for understanding face processing as a special case of social categorization. Below, we discuss each of these implications in turn.

\subsection{Implications for the Face Recognition Model}

As noted, the current findings broadly support Bruce and Young's (1986) face recognition model, in that they provide evidence for equally easy processing of group memberships and personal identity in faces. These findings, however, also present an avenue for extending the scope of that model. The face recognition model in its current guise is explicitly a model of personal identity recognition, in that it attempts to explain the sequence of cognitive-perceptual operations from detecting a face in the environment, through the construction of a representation of that face, to a final person-level identification and naming of that face. Our results suggest, however, that it could be transformed into a broader model 
of both person and group identification of faces, by relaxing the constraints that restrict it to the person-level. In particular, the assumption that the codes subserve person-level judgments could be relaxed, such that each could have a group-level equivalent (see Table 1). At present, only the visually derived semantic code is thought to result in the extraction of group information from faces directly, but our results suggest that each code could subserve both group and person-level operations, as these are simply different levels of representation, rather than different types of information per se (see Kramer, Young, Day \& Burton, 2017, for a similar suggestion). In this way, a perceiver could access the same pictorial, structural, identity-specific semantic, and name codes to come to an identification of the group membership of a face.

The pictorial code for person recognition represents an instance of that person's face, but there is no reason why it could not also represent an instance of a group face, as the chunked group task from Experiment 2 - in which each stimulus item represents an instance of an ethnic group - makes clear. The structural code for person recognition represents the structure of a person's face across instances, despite changes in such things as lighting, angle, situation, expressions. In the same way, the structural code for group recognition would represent the structure of a group face across instances, again controlling for these extraneous sources of variability. The identity-specific semantic code for person recognition represents the information derived from identifying the person to whom a face belongs. Similarly, the identity-specific semantic code for group recognition would represent information derived from identifying the group membership of a face, including previously formed impressions of that group, the likely behavioral orientations associated with members of that group, the likely geographic origin of that face, and so forth. Finally, the name code for group recognition, like that for person recognition, would simply encode the name of the group (for example, 'younger person' or 'Caucasian person'). Extending the scope of the face 
recognition model in this way would allow for a better understanding of the way in which human beings use both group and person information from faces when navigating their social worlds.

\subsection{Implication for the Continuum Model of Impression Formation}

As noted, the current findings challenge key aspects of Fiske and Neuberg's (1990) continuum model of impression formation, and the motivated tactician perspective (Fiske \& Taylor, 1991) in which it is embedded: that group memberships are easier to process than personal identity and that they are accessed earlier in the processing stream than personal identity. This challenge does not negate the model, however, but, as with the face recognition model (Bruce \& Young, 1986) allows for its scope to be extended. Fiske and Neuberg (1990) state that "a target's physical appearance often cues stereotypes", which are evident on the basis of "easily perceived physical features." The current results suggest that this can be extended such that "a target's physical appearance often cues [person impressions]", which are also evident on the basis of "easily perceived physical features.” In so doing, the continuum model would come to encapsulate various person-level phenomena, including, in particular processes of interpersonal transference and person-to-group induction. Interpersonal transference is the process of invoking a representation of a significant other when forming an impression of a novel target (but it can also refer to the process of invoking any person-level representation in impression formation; Andersen \& Berk, 1998; Andersen

\& Cole, 1990; Andersen, Glassman, Chen \& Cole, 1995; Andersen, Reznik \& Manzella, 1996). If person information can be automatically and easily extracted from a face, as our results suggest, then there is no reason to restrict the early stages of the continuum model to group information alone. As such, either group-level stereotypes or person-level impressions could be activated early in impression formation, and these could then frame the integration process, with different outcomes for the impression that is subsequently formed. Specifically, 
identifying a face in the environment as 'Clint Eastwood', for example, would result in the characteristics associated with Clint Eastwood being attributed to the target, after which the continuum model's confirmation, recategorization, and piecemeal integration stages would be framed around this initial impression.

Similarly, our finding that person information can be quickly extracted very early in the processing stream also allows the continuum model of impression formation to be expanded to encapsulate the process of person-to-group induction (Hamburger, 1994). Person-to-group induction refers to the process of using information about an individual group member to make inferences about the group to which they belong as a whole. If it is possible, as our results suggest, to access person information from faces very early in the processing stream, then, as with interpersonal transference, this person information can come to frame subsequent processes. And, if person impressions and group stereotypes are subserved by the same cognitive processes, as Skorich and Mavor (2013; Mavor, 2004) argue in particular, the current findings suggest that stereotypes could form on the basis of the characteristics of individual group members who have been recognized on the basis of their unique facial features. More broadly, then, combining the current findings with those of Skorich and Mavor (2013) results in transforming the continuum model from a model of impression formation into a model of both impression formation and stereotype formation, with both group and person information in faces potentially coming into play at the early stages. In this way, the current results serve to integrate previously disparate models of impression formation and stereotype formation under a single explanatory framework.

\subsection{Social Categorization and Face Processing}

By extending the face recognition (Bruce \& Young, 1986) and continuum models (Fiske \& Neuberg, 1990) to capture both group and person processes, the current findings pose the question as to what processes are involved in shifting a perceiver between group- 
level and person-level perception. As the results of Experiment 4 indicate, the same face instance can elicit either person-level or group-level recognition/identification, because every face contains both of these kinds of information, and both can be easily accessed relatively immediately. Experiment 4 also provides some answers to this question, as its results indicate that the prototypicality of a face exemplar is associated with the relative automaticity with which the entity of which it is part is activated. Prototypicality was operationalized in that experiment in a way consistent with its conceptualization within self-categorization theory (Turner et al., 1987, 1994) — such that an exemplar will be perceived as prototypical to the degree that it is most similar to other exemplars within the category of which it is a member and most different from exemplars within a comparison category. In the case of group-level face processes, the categories are group memberships such as sex, age, and ethnic background. In the case of person-level face processes, the categories are the individual identities and the category 'members' are the instances of that person's face across situations, occasions, different facial expressions and so on (Mavor, 2004; Skorich \& Mavor, 2013). As such, the structural descriptions of Bruce and Young's (1986) face recognition model can be seen as categories, and the pictorial depictions can be seen as the category members prior to integration.

Bringing together face recognition and the process of self-categorization in this way provides a powerful model with which to explain the way in which a perceiver will shift dynamically between person-level and group-level processing. Self-categorization theory (Turner et al., 1987, 1994) argues that perceivers will activate social categories at different levels of abstraction as a function of an interaction between perceiver readiness and fit (Blanz, 1999; Oakes, 1987). Perceiver readiness refers to the perceiver's goals, motivations, background theories, and general processing tendencies on entering a situation, as well as to the relative accessibility of categories in memory. Fit has a structural and normative 
component, where structural fit refers to the covariation between stimuli in the perceiver's current frame of reference and any number of potential categorization schemes, while normative fit refers to the degree to which that covariation is in the expected direction. Given a perceiver's particular state of readiness to process a face at the person-level or the grouplevel and the context-dependent structural relations between face exemplars in the perceiver's current frame of reference, then, a particular face categorization scheme will become activated, and, importantly, this scheme will be either at the group or person level. In Experiment 4, for example, perceiver readiness was given by our instructions to make either a group-level decision of old versus young or to make a person-level decision of Clint Eastwood versus Sean Connery, which gave participants a goal of detecting specific aspects of each exemplar presented to them. Structural fit was present in that experiment in the form of the prototypicality of each face exemplar, such that the structural relations between the categories old and young or Clint Eastwood and Sean Connery and the face exemplars were more readily apparent when those exemplars were more prototypical of those categories. In this way, the ease with which participants were able to make their group or person-level decisions was determined by both their own processing tendencies and goals and the structure of the stimulus frame. As such, these results provide initial support for the utility of a selfcategorization understanding of the dynamic activation/extraction of group or person information from faces.

\subsection{Limitations and Future Directions}

While the current findings provide important insights about face processing and social categorization, the experiments reported here do not come without some limitations. First, it was necessary to choose specific groups and specific persons in order to operationalize the level of representation of the task, which could limit the generalizability of these findings. It may be that the processes involved in face recognition/identification are different for different 
social categories and also for different pairs of individuals. We did, however, attempt to control for any such possible differences by measuring participants' familiarity with each of the groups and persons, and also by exploring different social categories and different individuals across the experiments reported here. In future, it would be useful to explore the same effects with a number of different group memberships - including sexual orientation (Rule et al., 2009) and political affiliation (Peterson et al., 2018) — and with a number of different individuals, particularly females, to ensure that the current results generalize to person and group information in faces in general.

In addition, the group-level codes that we suggest may exist if the face recognition model were extended need to be empirically validated. Specifically, it would be useful to explore the ways in which structural descriptions of faces are similar and different at the group and person levels, and whether these elicit the same types of identity-specific semantic content at each of these levels. Similarly, our suggestion that the self-categorization process is a key determinant of the level of face processing needs to be examined more extensively than they are in Experiment 4. This could involve manipulating structural and normative fit in faces directly, in paradigms suitable for that purpose, such as the 'who-said-what' paradigm (Taylor, Fiske, Etcoff \& Ruderman, 1978; see also Spears et al., 1999). Finally, we believe it would be worthwhile to explore impression formation and stereotype formation processes as outlined by way of an extended version of the continuum model (Fiske \& Neuberg, 1990), with particular reference to the way in which the initial extraction of person or group information from a face frames the subsequent impression.

\subsection{Conclusion}

In this paper, we explored the speed with which perceivers extract group and person information from faces. Across the four experiments we found no evidence for a processing advantage of either group or person information. Instead, chunked processing, featural 
processing, familiarity, and prototypicality were found to determine the speed of face processing, for both the group and person levels. We hope these findings provide the impetus for expanding existing models of face recognition and person perception, and, especially, for integrating previously disparate understandings of group and person processes in general. We see particular promise in conceptualizing face recognition — whether at the group-level or the person-level — as a special case of social categorization, such that the dynamic processes associated with moment-to-moment perception of a face can be better understood going forward. 


\section{List of Figures}

Figure 1. Examples of Johnny Depp and Brad Pitt Stimuli from Experiment 1.

Figure 2. Mean response latency across the three conditions of Experiment 1. Note: Error bars represent standard deviations.

Figure 3. Examples of chunked group stimuli from Experiment 2.

Figure 4. Mean response latency, adjusted for level of relative familiarity, across group and person conditions, as a function of the number of chunks. Note: Error bars represent standard deviations.

Figure 5. Examples of George Clooney and Bruce Willis stimuli from Experiment 3.

Figure 6. Mean response latency across group and person cue-present and cue-absent conditions. Note: Error bars represent standard deviations.

Figure 7. Examples of old and young Sean Connery and old and young Clint Eastwood stimuli from Experiment 4.

Figure 8. Mean response latency across group and person conditions, as a function of prototypicality. Note: Error bars represent standard deviations. 


\section{References}

Alimena, B. S. (1962). A method of determining unbiased distribution in the Latin square. Psychometrika, 27(3), 315-317.

Andersen, S. M., \& Berk, M. S. (1998). The social-cognitive model of transference:

Experiencing past relationships in the present. Current Directions in Psychological Science, 7(4), 109-115.

Andersen, S. M., \& Cole, S. W. (1990). " Do I know you?": The role of significant others in general social perception. Journal of Personality and Social Psychology, 59(3), 384.

Andersen, S. M., Glassman, N. S., Chen, S., \& Cole, S. W. (1995). Transference in social perception: The role of chronic accessibility in significant-other representations. Journal of Personality and Social Psychology, 69(1), 41.

Andersen, S. M., Reznik, I., \& Manzella, L. M. (1996). Eliciting facial affect, motivation, and expectancies in transference: Significant-other representations in social relations. Journal of Personality and Social Psychology, 71(6), 1108.

Baron-Cohen, S. (2005). The Empathizing System: A revision of the 1994 model of the Mindreading System. In B. Ellis \& D. Bjorklund (Eds.), Origins of the Social Mind: Evolutionary Psychology and Child Development (pp.468-492). New York: Guildford Press.

Baron-Cohen, S., Jolliffe, T., Mortimore, C., \& Robertson, M. (1997). Another advanced test of theory of mind: Evidence from very high functioning adults with autism or Asperger syndrome. Journal of Child Psychology and Psychiatry, 38(7), 813-822.

Bartlett, J. C., \& Searcy, J. (1993). Inversion and configuration of faces. Cognitive psychology, 25(3), 281-316.

Bentin, S., \& Deouell, L. Y. (2000). Structural encoding and identification in face processing: ERP evidence for separate mechanisms. Cognitive neuropsychology, 17(1-3), 35-55. 
Blair, I. V., Judd, C. M., \& Fallman, J. L. (2004). The Automaticity of Race and Afrocentric Facial Features in Social Judgments. Journal of Personality and Social Psychology, 87(6), $763-778$.

Blanz, M. (1999). Accessibility and fit as determinants of the salience of social categorizations. European Journal of Social Psychology, 29(1), 43-74.

Bodenhausen, G. V., Macrae, C. N., \& Sherman, J. W. (1999). On the dialectics of discrimination: dual processes in social stereotyping. In S. Chaiken \& Y. Trope. (Eds.), Dual Process Theories in Social Psychology. New York, NY: Guildford Press.

Broadbent, D. E. (1975). The magic number seven after fifteen years. In A. Kennedy \& A. Wilkes (Eds.), Studies in Long-Term Memory (pp. 3-18). London: Wiley.

Brown, E., \& Perrett, D. I. (1993). What gives a face its gender? Perception, 22(7), 829-840. Bruce, V. (1983). Recognizing faces. Philosophical Transactions of the Royal Society of London. B, Biological Sciences, 302(1110), 423-436.

Bruce, V., Ellis, H. D., Gibling, F., \& Young, A. (1987). Parallel processing of the sex and familiarity of faces. Canadian Journal of Psychology/Revue canadienne de psychologie, 41(4), 510.

Bruce, V., \& Young, A. (1986). Understanding face recognition. British journal of psychology, 77(3), 305-327.

Bruce, V., \& Young, A. W. (2012). Face perception. Psychology Press.

Burton, A. M., Bruce, V., \& Dench, N. (1993). What's the difference between men and women? Evidence from facial measurement. Perception, 22(2), 153-176.

Burton, A. M., Jenkins, R., Hancock, P. J. B., \& White, D. (2005). Robust representations for face recognition: the power of averages. Cognitive Psychology, 51. 256-284.

Caldara, R., Jermann, F., Arango, G. L., \& Van der Linden, M. (2004). Is the N400 categoryspecific? A face and language processing study. Neuroreport, 15(17), 2589-2593. 
Cloutier, J., \& Macrae, C. N. (2007). Who or what are you?: Facial orientation and person construal. European Journal of Social Psychology, 37(6), 1298-1309.

Cloutier, J., Mason, M. F., \& Macrae, C. N. (2005). The perceptual determinants of person construal: Reopening the social-cognitive toolbox. Journal of Personality and Social Psychology, 88(6), 885.

Cohen, J. (1988). Statistical Power Analysis for the Behavioral Sciences, $2^{\text {nd }}$ Edition. Hillsdale, NJ: Lawrence Erlbaum.

Cohen, J. (1992). Statistical power analysis. Current Directions in Psychological Science, 1(3). 98-101.

Eimer, M. (2000a). The face-specific N170 component reflects late stages in the structural encoding of faces. Neuroreport, 11(10), 2319-2324.

Eimer, M. (2000b). Event-related brain potentials distinguish processing stages involved in face perception and recognition. Clinical neurophysiology, 111(4), 694-705.

Fiske, S. T., Lin, M., \& Neuberg, S. (1999). The continuum model. Dual-process theories in social psychology, 321-254.

Fiske, S. T., \& Neuberg, S. L. (1990). A continuum of impression formation, from categorybased to individuating processes: Influences of information and motivation on attention and interpretation. In Advances in experimental social psychology (Vol. 23, pp. 1-74). Academic Press.

Fiske, S. T., \& Taylor, S. E. (1991). Social cognition. Mcgraw-Hill Book Company.

Freeman, J. B., Ambady, N., \& Holcomb, P. J. (2010). The face-sensitive N170 encodes social category information. Neuroreport, 21(1), 24.

Hamburger, Y. (1994). The contact hypothesis reconsidered: Effects of the atypical outgroup member on the outgroup stereotype. Basic and Applied Social Psychology, 15(3), 339358. 
Hamilton, D. L., \& Sherman, S. J. (1996). Perceiving persons and groups. Psychological Review, 103. 336-355.

Hancock, P. J. B., Bruce, V., \& Burton, A. M. (2000). Recognition of unfamiliar faces. Trends in Cognitive Sciences, 4(9). 330-337.

Hehman, E., Volpert, H. I., \& Simons, R. F. (2014). The N400 as an index of racial stereotype accessibility. Social Cognitive and Affective Neuroscience, 9(4), 544-552.

Huang, W., Wu, X., Hu, L., Wang, L., Ding, Y., \& Qu, Z. (2017). Revisiting the earliest electrophysiological correlate of familiar face recognition. International Journal of Psychophysiology, 120, 42-53.

Ito, T. A., \& Urland, G. R. (2003). Race and gender on the brain: electrocortical measures of attention to the race and gender of multiply categorizable individuals. Journal of Personality and Social Psychology, 85(4), 616.

Ito, T. A., \& Urland, G. R. (2005). The influence of processing objectives on the perception of faces: An ERP study of race and gender perception. Cognitive, Affective, and Behavioral Neuroscience, 5(1), 21-36.

Kramer, R. S., Young, A. W., Day, M. G., \& Burton, A. M. (2017). Robust social categorization emerges from learning the identities of very few faces. Psychological Review, 124(2), 115.

Kubota, J. T., \& Ito, T. (2017). Rapid race perception despite individuation and accuracy goals. Social Neuroscience, 12(4), 468-478.

Linville, P. W., Fischer, G. W., \& Yoon, C. (1996). Perceived covariation among the features of ingroup and outgroup members: The outgroup covariation effect. Journal of Personality and Social Psychology, 70(3), 421.

Macrae, C. N., \& Martin, D. (2007). A boy primed Sue: Feature-based processing and person construal. European Journal of Social Psychology, 37(5), 793-805. 
Macrae, C. N., Quinn, K. A., Mason, M. F., \& Quadflieg, S. (2005). Understanding others: The face and person construal. Journal of Personality and Social Psychology, 89(5), 686.

Mason, M. F., \& Macrae, C. N. (2004). Categorizing and individuating others: The neural substrates of person perception. Journal of Cognitive Neuroscience, 16(10), 1785-1795.

Martin, D., \& Macrae, C. N. (2007). A face with a cue: Exploring the inevitability of person categorization. European Journal of Social Psychology, 37(5), 806-816.

Mavor, K. I. (August, 2004). Apples, oranges, and... tomatoes? Comparing groups, persons, and other social entities and the identities that go with them. $12^{\text {th }}$ Brisbane Symposium on Social Identity, University of Queensland, Brisbane, Australia.

McGarty, C. (1999). Categorization in Social Psychology. London, UK: Sage.

Miller, G. A. (1956). The magic number seven plus or minus two: Some limits on our capacity for processing information. Psychological review, 63, 91-97.

Monroe, B. M., Koenig, B. L., Wan, K. S., Laine, T., Gupta, S., \& Ortony, A. (2018). Reexamining dominance of categories in impression formation: A test of dual-process models. Journal of Personality and Social Psychology, 115(1), 1.

Oakes, P. J., Haslam, S. A., \& Turner, J. C. (1994). Stereotyping and Social Reality. Oxford, UK: Blackwell.

O’Toole, A. J., Abdi, H., Deffenbacher, K. A., \& Valentin, D. (1993). Low-dimensional representation of faces in higher dimensions of the face space. JOSA A, 10(3), 405-411.

Palermo, R., Jeffery, L., Lewandowsky, J., Fiorentini, C., Irons, J. L., Dawel, A., ... \& Rhodes, G. (2018). Adaptive face coding contributes to individual differences in facial expression recognition independently of affective factors. Journal of Experimental Psychology: Human Perception and Performance, 44(4), 503. 
Peterson, J. C., Jacobs, C., Hibbing, J., \& Smith, K. (2018). In your face: Emotional expressivity as a predictor of ideology. Politics and the Life Sciences, 37(1), 53-67.

Quinn, K. A., Mason, M. F., \& Macrae, C. N. (2009). Familiarity and person construal: Individuating knowledge moderates the automaticity of category activation. European Journal of Social Psychology, 39(5), 852-861.

Quinn, K. A., Mason, M. F., \& Macrae, C. N. (2010). When Arnold is “The Terminator”, We No Longer See Him as a Man. Experimental Psychology.

Reynolds, K. J., \& Oakes, P. J. (2000). Variability in impression formation: Investigating the role of motivation, capacity, and the categorization process. Personality and Social Psychology Bulletin, 26(3), 355-373.

Rosch, E. (1978). Principles of categorization. In E. Rosch \& B. B. Lloyd (Eds.), Cognition and categorization (pp. 27-48). Hillsdale, NJ: Erlbaum.

Rossion, B. (2002). Is sex categorization from faces really parallel to face recognition? Visual Cognition, 9(8). 1003-1020.

Rule, N. O., Ambady, N., \& Hallett, K. C. (2009). Female sexual orientation is perceived accurately, rapidly, and automatically from the face and its features. Journal of Experimental Social Psychology, 45(6), 1245-1251.

Rule, N. O., \& Sutherland, S. L. (2017). Social categorization from faces: Evidence from obvious and ambiguous groups. Current Directions in Psychological Science, 26(3), 231-236.

Searcy, J. H., \& Bartlett, J. C. (1996). Inversion and processing of component and spatialrelational information in faces. Journal of Experimental Psychology: Human Perception and Performance, 22(4), 904.

Senholzi, K. B., \& Ito, T. A. (2013). Structural face encoding: how task affects the N170's sensitivity to race. Social Cognitive and Affective Neuroscience, 8(8), 937-942. 
Sergent, J. (1986). Microgenesis of face perception. In Aspects of face processing (pp. 1733). Springer, Dordrecht.

Sherman, J. W., \& Frost, L. A. (2000). On the encoding of stereotype-relevant information under cognitive load. Personality and Social Psychology Bulletin, 26. 26-34.

Sherman, J. W., Macrae, C. N., \& Bodenhausen, G. V. (2000). Attention and stereotyping: cognitive constraints on the construction of meaningful social impressions. In W. Stroebe \& M. Hewstone (Eds.), European Review of Social Psychology (Vol. 11, pp. 145-175). Chichester, UK: Wiley.

Skorich, D. P., \& Mavor, K. I. (2013). Cognitive load privileges memory-based over datadriven processing, not group-level over person-level processing. British Journal of Social Psychology, 52(3), 469-488.

Smithson, M. (2002). Confidence intervals (Vol. 140). Sage Publications.

Spears, R., Haslam, S. A., \& Jansen, R. (1999). The effect of cognitive load on social categorization in the category confusion paradigm. European Journal of Social Psychology, 29, 621-640.

Tanaka, J. W. (2001). The entry point of face recognition: Evidence for face expertise. Journal of Experimental Psychology: General, 130(3). 534-543.

Taylor, S. E., Fiske, S. T., Etcoff, N. L., \& Ruderman, A. J. (1978). Categorical and contextual bases of person memory and stereotyping. Journal of Personality and Social Psychology, 36(7), 778-793.

Todorov, A., Olivola, C. Y., Dotsch, R., \& Mende-Siedlecki, P. (2015). Social attributions from faces: Determinants, consequences, accuracy, and functional significance. Annual review of psychology, 66 . 
Turner, J. C., Hogg, M. A., Oakes, P. J., Reicher, S. D., \& Wetherell, M. S.

(1987). Rediscovering the Social Group: A Self-Categorization Theory. Oxford: Basil Blackwell.

Turner, J. C., Oakes, P. J., Haslam, S. A., \& McGarty, C. (1994). Self and collective:

Cognition and social context. Personality and Social Psychology Bulletin, 20, 454454.

Valentine, T., \& Ferrara, A. (1991). Typicality in categorization, recognition and identification: Evidence from face recognition. British Journal of Psychology, 82. 87102.

Yan, X., Young, A. W., \& Andrews, T. J. (2017). The automaticity of face perception is influenced by familiarity. Attention, Perception, \& Psychophysics, 79(7), 2202-2211.

Young, A. W., \& Bruce, V. (2011). Understanding person perception. British journal of psychology, 102(4), 959-974. 


\begin{tabular}{lll}
\hline Codes & Person Identification & Group Identification \\
\hline Pictorial & Instance of person & Instance of group \\
& & \\
Structural & $\begin{array}{l}\text { Representation of structure of } \\
\text { individual face, despite changes in } \\
\text { lighting, angle, situation etc. }\end{array}$ & $\begin{array}{l}\text { Representation of structure of } \\
\text { group face, despite changes in } \\
\text { lighting, angle, individual etc. }\end{array}$ \\
& $\begin{array}{l}\text { Information derived from } \\
\text { recognition }\end{array}$ & $\begin{array}{l}\text { Information derived from } \\
\text { recognition }\end{array}$ \\
Identity-specific & & \\
& & Group's name \\
Name & Individual's name &
\end{tabular}

Table 1. Face recognition codes for person identification, with suggested group identification equivalents. 

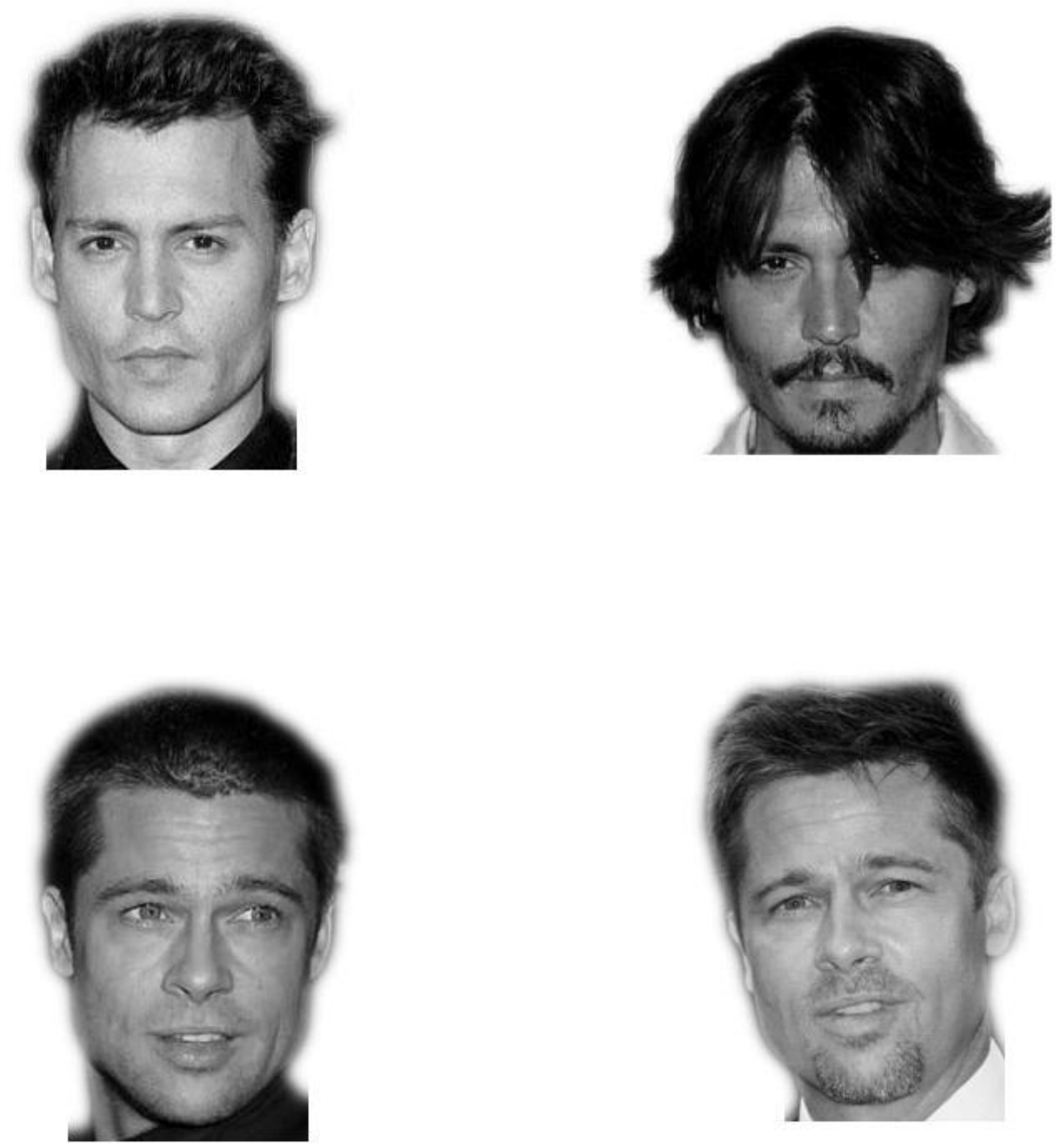

Figure 1. Examples of Johnny Depp and Brad Pitt stimuli from Experiment 1. 


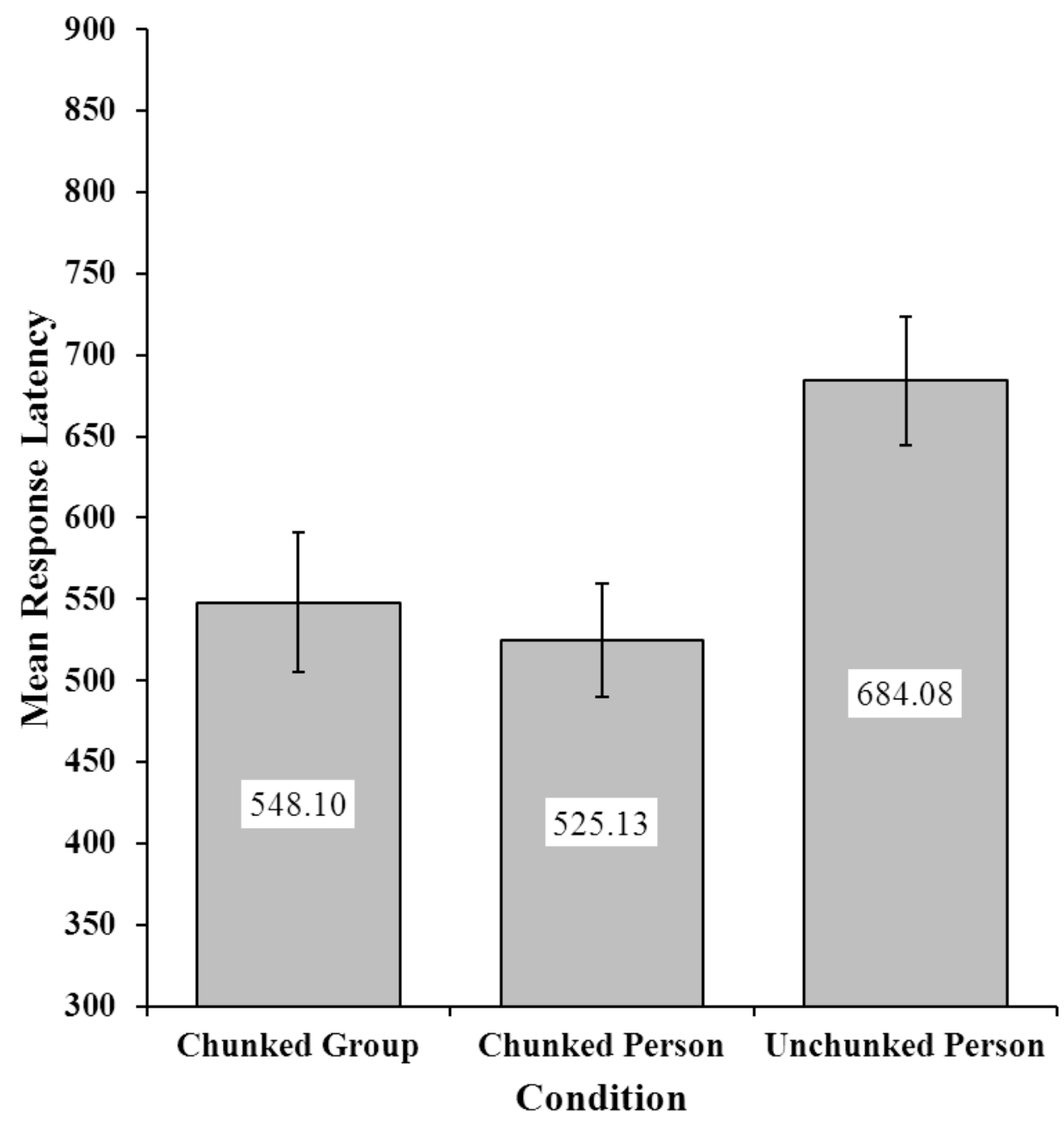

Figure 2. Mean response latency across the three conditions of Experiment 1. Note: Error bars represent standard deviations. 

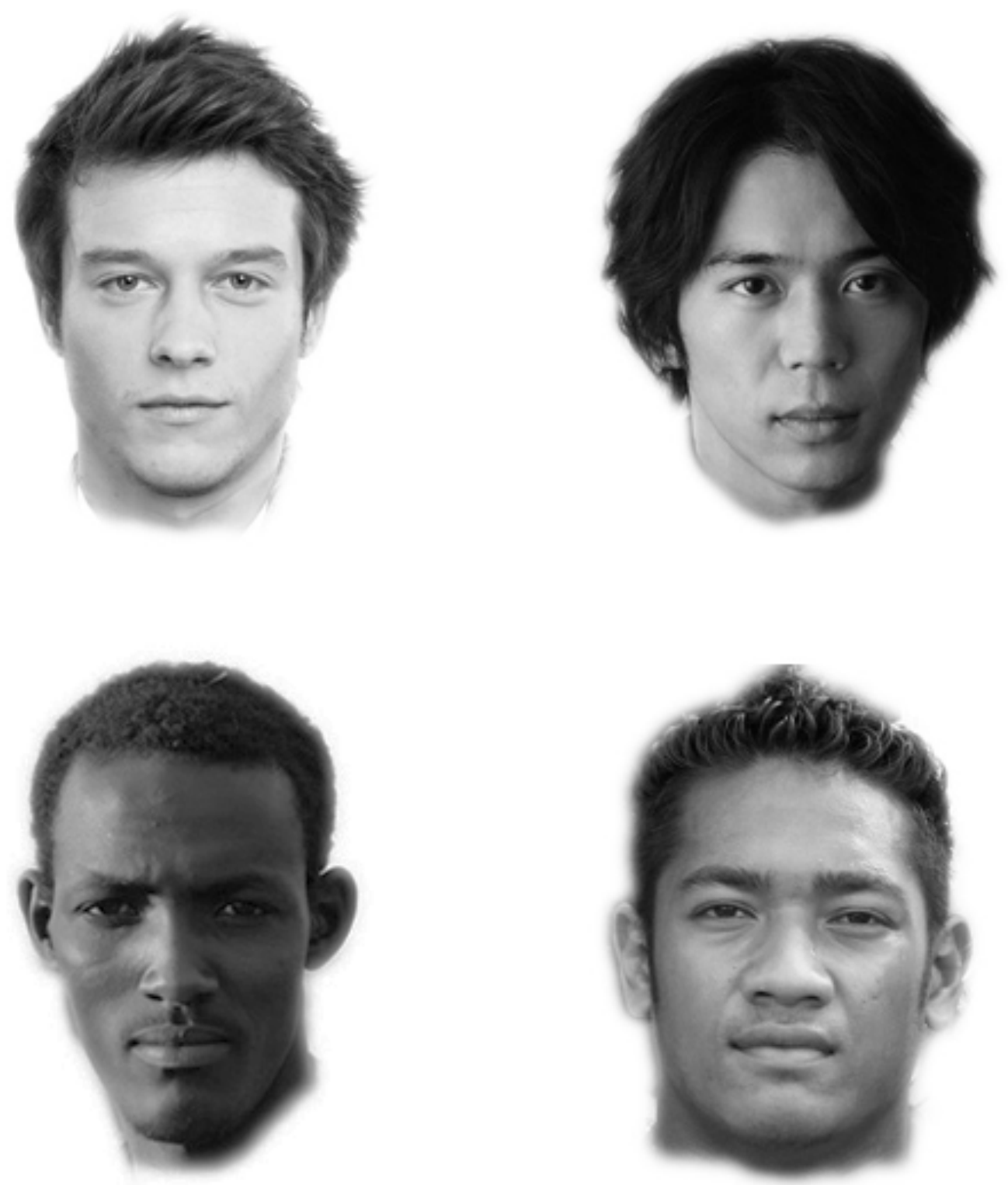

Figure 3. Examples of chunked group stimuli in Experiment 2. 


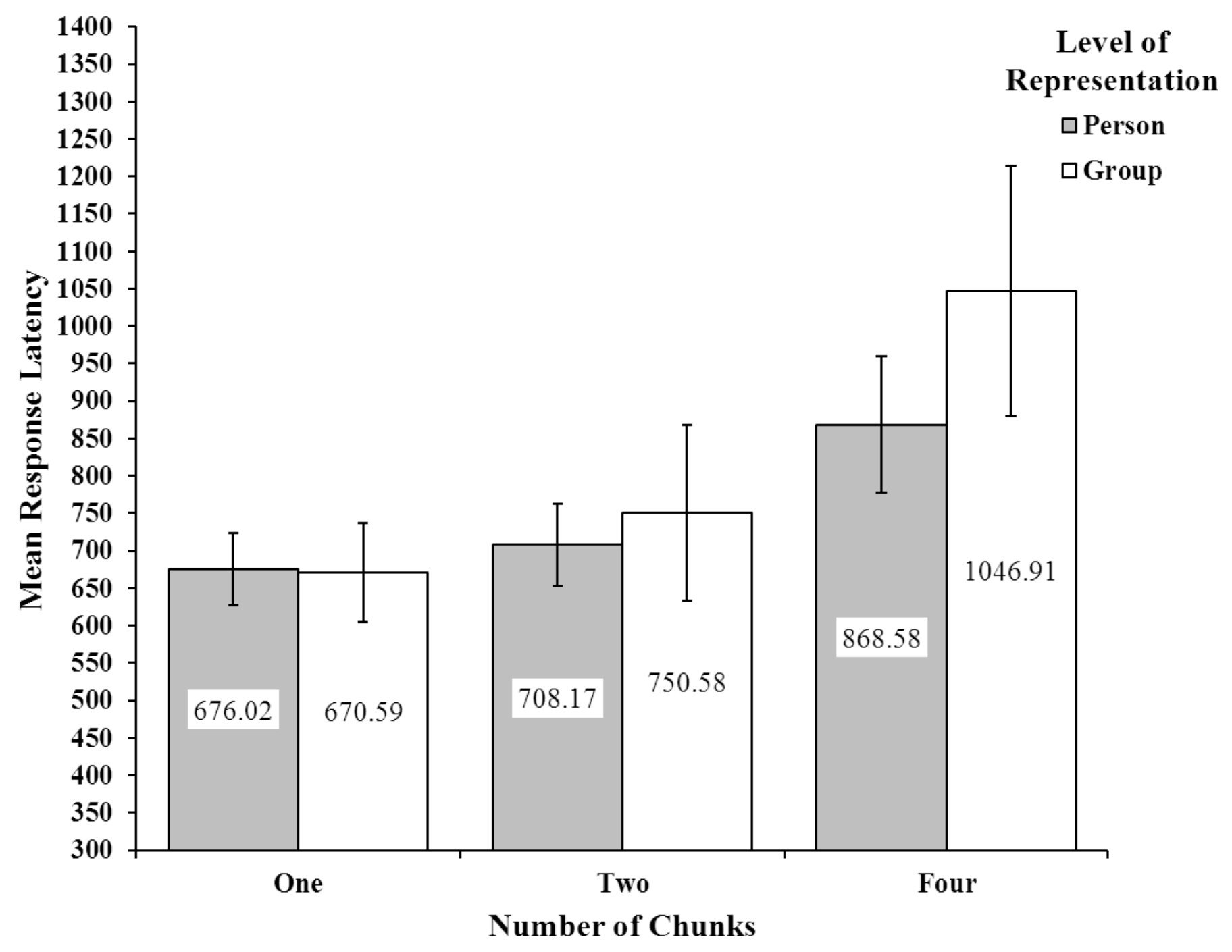

Figure 4. Mean response latency, adjusted for level of relative familiarity, across group and person conditions, as a function of the number of chunks. Note: Error bars represent standard deviations. 

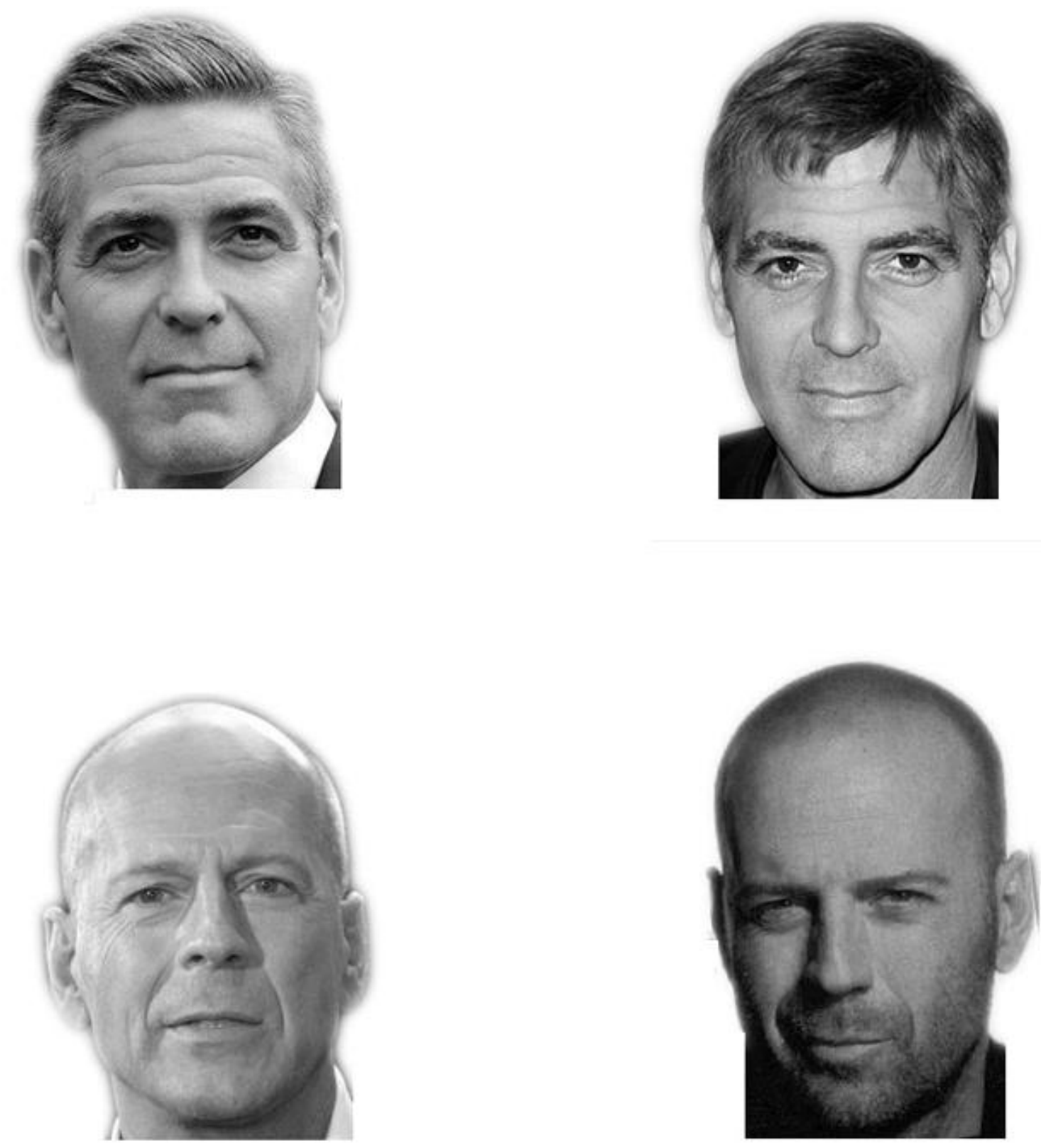

Figure 3. Examples of George Clooney and Bruce Willis stimuli from Experiment 3. 


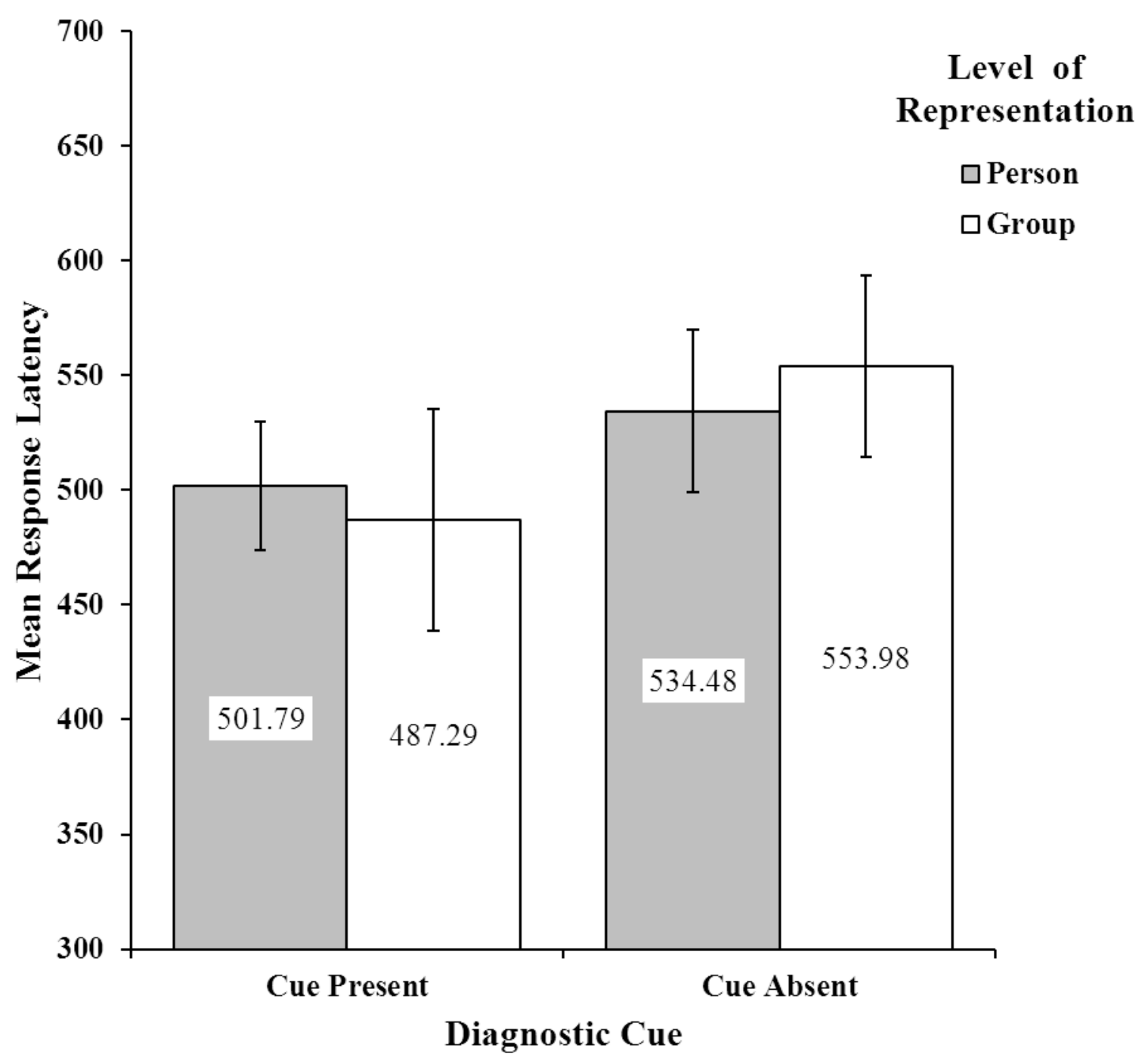

Figure 6. Mean response latency across group and person cue-present and cue-absent conditions. Note: Error bars represent standard deviations. 

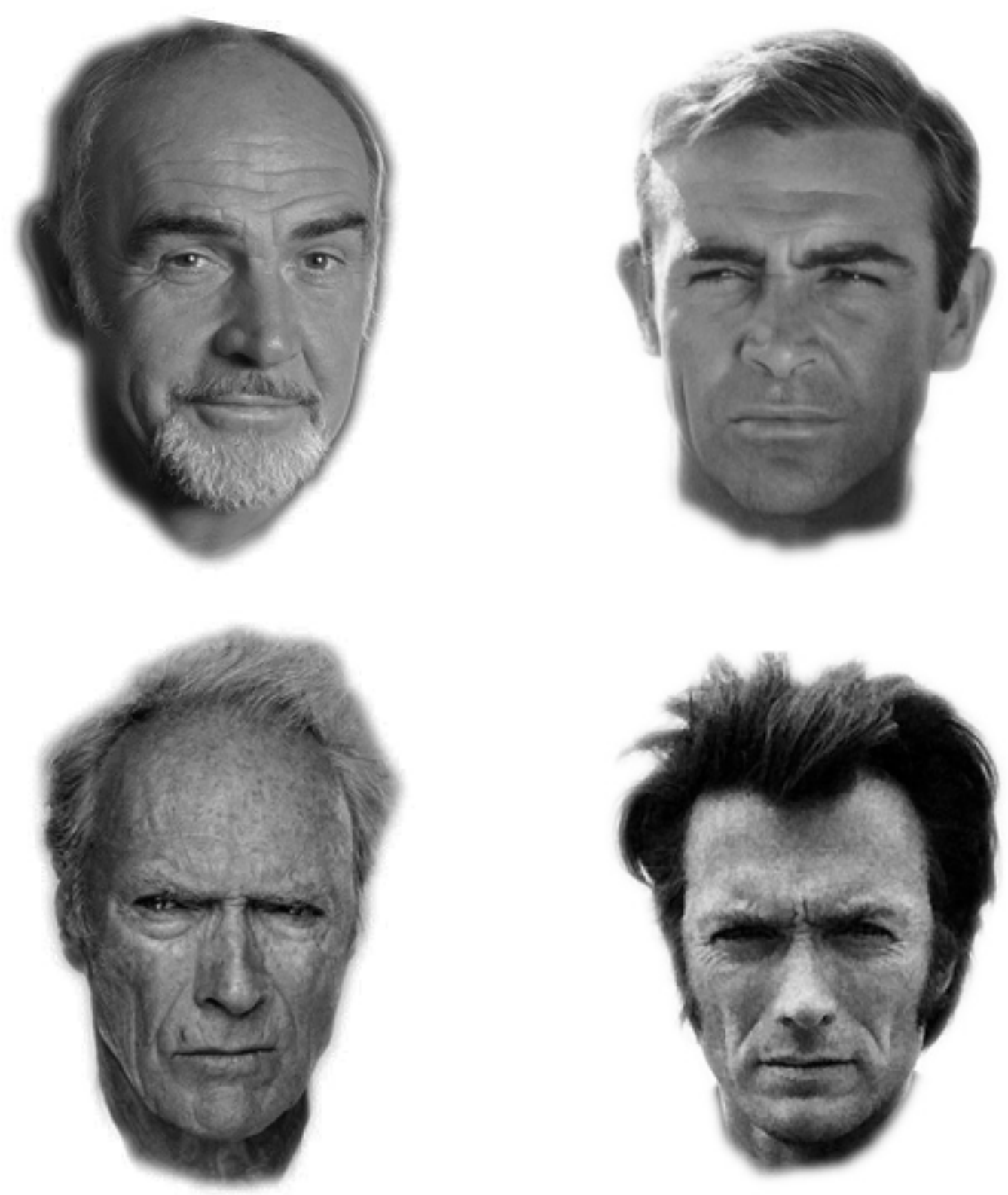

Figure 7. Examples of old and young Sean Connery and old and young Clint Eastwood stimuli from Experiment 4. 


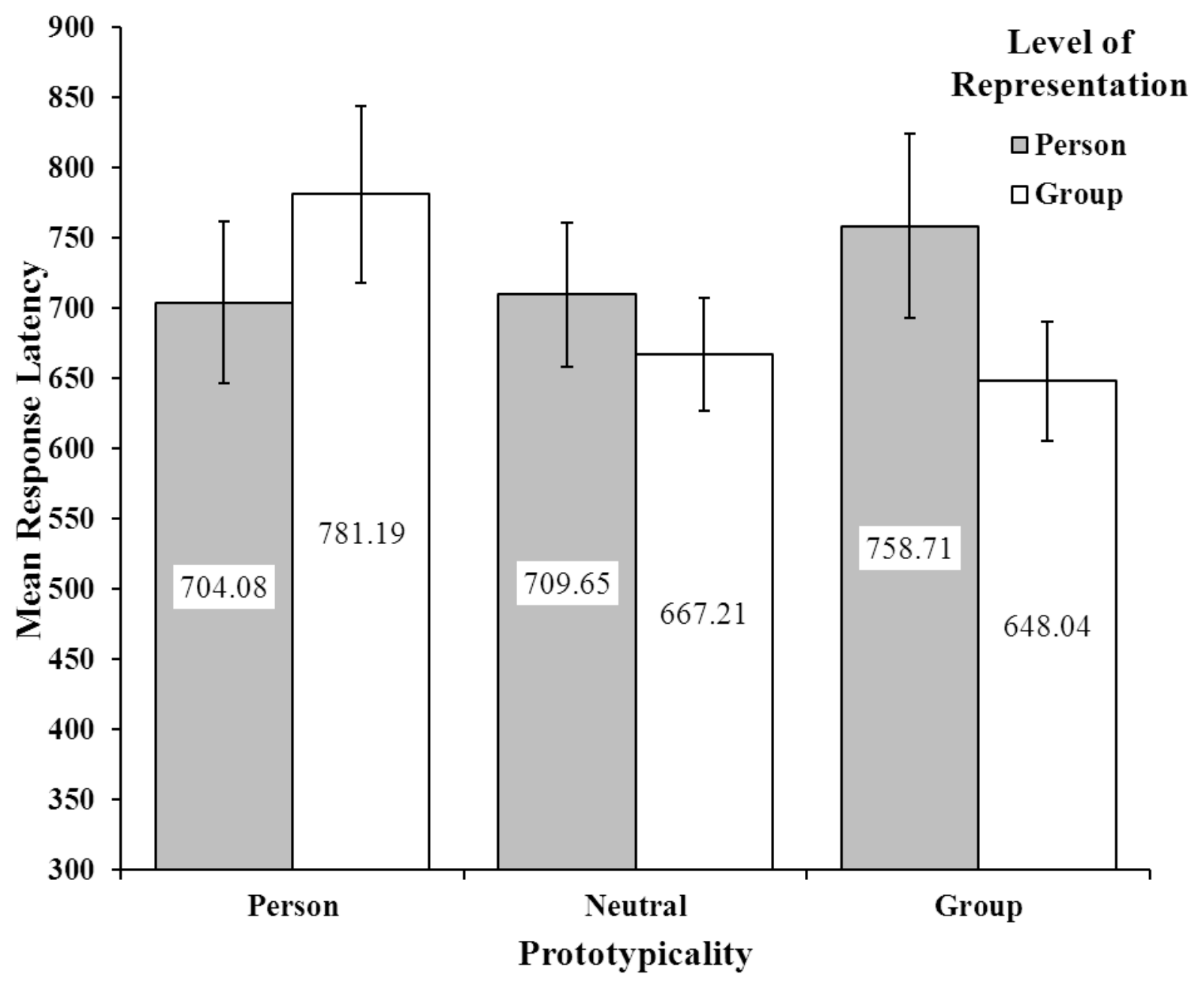

Figure 8. Mean response latency across group and person conditions, as a function of prototypicality. Note: Error bars represent standard deviations. 